\title{
APPROXIMATE CONTROLLABILITY FOR NAVIER-STOKES EQUATIONS IN 3D RECTANGLES UNDER LIONS BOUNDARY CONDITIONS
}

\author{
DUY PHAN AND SÉRGIO S. RODRIGUES
}

\begin{abstract}
The 3D Navier-Stokes system, under Lions boundary conditions, is proven to be approximately controllable provided a suitable saturating set does exist. An explicit saturating set for $3 \mathrm{D}$ rectangles is given.

MSC2010: 93B05, 35Q30, 93C20.

Keywords: Navier-Stokes equations; approximate controllability; saturating set.
\end{abstract}

\section{INTRODUCTION}

We consider the incompressible 3D Navier-Stokes system in $(0, T) \times \Omega$, under Lions boundary conditions,

$$
\begin{array}{rlrl}
\partial_{t} u+\langle u \cdot \nabla\rangle u-\nu \Delta u+\nabla p+h & =\eta, & \operatorname{div} u & =0, \\
\left.\left(\begin{array}{c}
u \cdot \mathbf{n} \\
\operatorname{curl} u-((\operatorname{curl} u) \cdot \mathbf{n}) \mathbf{n}
\end{array}\right)\right|_{\partial \Omega} & =\left(\begin{array}{l}
0 \\
0
\end{array}\right), & u(0, x)=u_{0}(x),
\end{array}
$$

where $\Omega \subset \mathbb{R}^{3}$ is a rectangle $\Omega=\left(0, L_{1}\right) \times\left(0, L_{2}\right) \times\left(0, L_{3}\right)$, whose boundary is denoted by $\partial \Omega$. As usual $u=\left(u_{1}, u_{2}, u_{3}\right)$ and $p$, defined for $\left(t, x_{1}, x_{2}, x_{3}\right) \in I \times \Omega$, are respectively the unknown velocity field and pressure of the fluid, $\nu>0$ is the viscosity, the operators $\nabla$ and $\Delta$ are respectively the well known gradient and Laplacian in the space variables $\left(x_{1}, x_{2}, x_{3}\right) \in \Omega$, $\langle u \cdot \nabla\rangle v$ stands for $\left(u \cdot \nabla v_{1}, u \cdot \nabla v_{2}, u \cdot \nabla v_{3}\right)$, div $u:=\sum_{i=1}^{3} \partial_{x_{i}} u_{i}$, the vector $\mathbf{n}$ stands for the outward unit normal vector to $\partial \Omega$, and $h$ is a fixed function. Finally, $\eta$ is a control at our disposal.

Lions boundary conditions (cf. [15, Section 6.9]) are a particular case of Navier boundary conditions. For works and motivations concerning Lions and Navier boundary conditions (in both $2 \mathrm{D}$ and $3 \mathrm{D}$ cases) we refer to [8, 13, 14, 21, 34, 35] and references therein.

1.1. The evolutionary system. We can rewrite system (11) as an evolutionary system

$$
\dot{u}+A u+B(u, u)+h=\eta, \quad u(0)=u_{0},
$$

in the subspace $H:=\left\{u \in L^{2}\left(\Omega, \mathbb{R}^{3}\right) \mid \operatorname{div} u=0\right.$ and $\left.\left.(u \cdot \mathbf{n})\right|_{\partial \Omega}=0\right\}$ of divergence free vector fields which are tangent to the boundary. We may suppose that $h$ and $\eta$ take their values in $H$ (otherwise we just take their orthogonal projections onto $H$ ). We consider $H$, endowed with the norm inherited from $L^{2}\left(\Omega, \mathbb{R}^{3}\right)$, as a pivot space, that is, $H=H^{\prime}$. Further we set the spaces

$$
\begin{aligned}
V & :=\left\{u \in H^{1}\left(\Omega, \mathbb{R}^{3} \mid u \in H\right\},\right. \\
\mathrm{D}(A) & :=\left\{u \in H^{2}\left(\Omega, \mathbb{R}^{3}\right)|u \in H, \quad \operatorname{curl} u-((\operatorname{curl} u) \cdot \mathbf{n}) \mathbf{n}|_{\partial \Omega}=0\right\}
\end{aligned}
$$

Above, for $u, v, w \in V$,

$$
\begin{array}{lr}
A: V \rightarrow V^{\prime}, & \langle A u, v\rangle_{V^{\prime}, V}:=\nu(\operatorname{curl} u, \operatorname{curl} v)_{L^{2}\left(\Omega, \mathbb{R}^{3}\right)}, \\
B: V \times V \rightarrow V^{\prime}, & \langle B(u, v), w\rangle_{V^{\prime}, V}:=-\int_{\Omega}(\langle u \cdot \nabla\rangle w) \cdot v \mathrm{~d} \Omega .
\end{array}
$$

It turns out that $\mathrm{D}(A)=\{u \in H \mid A u \in H\}$ is the domain of $A$. We will refer to $A$ as the Stokes operator, under Lions boundary conditions. Further, we have the continuous, dense, and compact inclusions $\mathrm{D}(A) \stackrel{\mathrm{d}, \mathrm{c}}{\longrightarrow} V \stackrel{\mathrm{d}, \mathrm{c}}{\longrightarrow} H$.

Denoting by $\Pi$ the orthogonal projection in $L^{2}\left(\Omega, \mathbb{R}^{3}\right)$ onto $H$, for $u, v \in \mathrm{D}(A)$ we may write $A u:=\Pi(\nu \Delta u)$, and $B(u, v):=\Pi(\langle u \cdot \nabla\rangle v)$. 
Further $A$ maps $V$ onto $V^{\prime}$, and the operator $A^{-1} \in \mathcal{L}(H)$ is compact. The eigenvalues of $A$, repeated accordingly with their multiplicity, form an increasing sequence $\left(\underline{\lambda}_{k}\right)_{k \in \mathbb{N}_{0}}$,

$$
0<\underline{\lambda}_{1} \leq \underline{\lambda}_{2} \leq \underline{\lambda}_{3} \leq \underline{\lambda}_{4} \leq \ldots,
$$

with $\underline{\lambda}_{k}$ going to $+\infty$ with $k$.

Remark 1.1. It is clear that the Stokes operator (3) is well defined, mapping $V$ into $V^{\prime}$. We also see that the bilinear operator (4) maps $V \times V$ into $V^{\prime}$, due to the estimate

$$
\begin{aligned}
\langle B(u, v), w\rangle_{V^{\prime}, V} & \leq \mathrm{C}_{1}|u|_{L^{6}\left(\Omega, \mathbb{R}^{3}\right)}|\nabla w|_{L^{2}\left(\Omega, \mathbb{R}^{9}\right)}|v|_{L^{3}\left(\Omega, \mathbb{R}^{3}\right)} \\
& \leq \mathrm{C}_{2}|u|_{H^{1}\left(\Omega, \mathbb{R}^{3}\right)}|w|_{H^{1}\left(\Omega, \mathbb{R}^{3}\right)}|v|_{H^{1}\left(\Omega, \mathbb{R}^{3}\right)}
\end{aligned}
$$

For further estimations on the bilinear operator we refer to [33, Section 2.3].

1.2. Saturating sets and approximate controllability. In the pioneering work [3] the authors introduced a method which led to the controllability of finite-dimensional Galerkin approximations of the 2D and 3D Navier-Stokes system, and to the approximate controllability of the 2D Navier-Stokes system, by means of low modes/degenerate forcing.

Hereafter $U \subseteq H$ will stand for a linear subspace of $H$, and we denote

$$
\mathcal{B}(a, b):=B(a, b)+B(b, a) .
$$

Definition 1.1. Let $\mathcal{C}=\left\{W_{k} \mid k \in\{1,2, \ldots, M\}\right\}$ and let $E$ be a finite-dimensional space so that $\mathcal{C} \subset E \subset U$. The finite-dimensional subspace $\mathcal{F}_{\mathrm{L}}(E) \subset U$ is given by

$$
\mathcal{F}_{\mathrm{L}}(E):=E+\operatorname{span}\{\mathcal{B}(a, b) \mid a \in \mathcal{C}, b \in E \text {, and }(B(a, a), B(b, b)) \in H \times H\} \cap U,
$$

Definition 1.2. A given finite subset $\mathcal{C}=\left\{W_{k} \mid k \in\{1,2, \ldots, M\}\right\} \subset U$ is said (L, $U$ )saturating if for the following sequence of subspaces $\mathcal{G}^{j} \subset U$, defined recursively by

$$
\mathcal{G}^{0}:=\operatorname{span} \mathcal{C}, \quad \mathcal{G}^{j+1}:=\mathcal{F}_{\mathrm{L}}\left(\mathcal{G}^{j}\right),
$$

we have that the union $\bigcup_{j \in \mathbb{N}} \mathcal{G}^{j}$ is dense in $H$.

In [4, Section 4] an explicit saturating set with 4 elements is presented for the 2D NavierStokes system under periodic boundary conditions.

Remark 1.2. In order to deal with different types of boundary conditions and domains the definitions of saturating set has been slightly changed/relaxed in several works. The definition of saturating set in [4, Section 4] is slightly different from Definition [1.2. But, we can prove (cf. [25, Section 6.1]) that the saturating set presented in [4] is also (L, $\mathrm{D}(A)$ )-saturating (cf. [25, Definition 2.2.1]). Actually, in [4] saturating sets are defined through the frequencies (say, indexes) of eigenfunctions of the Stokes operator, but we can rewrite the definition in terms of the eigenfunctions themselves.

We would like to refer also to the works [10,12,26, where the notion of saturating set was used to derive ergodicity for the Navier-Stokes system under degenerate stochastic forcing (compare the sequence of subsets $\mathcal{Z}_{n}$ in [12, Section 4] with the sequence of subsets $\mathcal{K}^{n}$ in [3, Section 8]).

In the pioneering work [3] the set $U$ in (1.2) is taken to be $\mathrm{D}(A)$, the same is done in [4,22, 23, 28. Later, in [20,24,25], $U$ is taken as $V$ in order to delal either with Navier-type boundary conditions or with internal controls supported in a small subset.

Often, for 2D Navier-Stokes equations and 1D Burgers equations, we have estimates for the bilinear term $B(\cdot, \cdot)$ which allow us to derive the well-posedness of the Cauchy problem, or that we can use to derive the controllability results. For example, the estimate

$$
\left|\langle B(z+y, y), z\rangle_{V^{\prime}, V}\right|_{\mathbb{R}} \leq \mathrm{C}_{1}|z|_{H}|z|_{V}|y|_{V}+\mathrm{C}_{1}|y|_{H}|y|_{V}|z|_{V}
$$

is used in [20] in the $1 \mathrm{D}$ and $2 \mathrm{D}$ settings, to derive approximate controllability results for the Navier-Stokes and Burgers equations. The same estimate

$$
\left|\langle B(z, y), z\rangle_{V^{\prime}, V}\right|_{\mathbb{R}} \leq \mathrm{C}_{1}|z|_{H}|z|_{V}|y|_{V}
$$


above can be used to prove the uniqueness of weak solutions for the corresponding systems. The estimate does not hold in the 3D case.

In [28, the method introduced in [3] is developed so the case where the well-posedness of the Cauchy problem is not known.

Definition 1.3. Given a finite dimensional space $E \subset U$. The finite-dimensional $\mathcal{F}_{\mathrm{B}}(E)$ is the largest linear subspace $F \subset U$ so that any $\eta_{1} \in F$ can be written as

$$
\eta_{1}=\eta-\sum_{j=1}^{k} \alpha_{j} B\left(\zeta^{j}\right),
$$

with $k \in \mathbb{N}_{0},\left(\eta, \zeta^{1}, \ldots, \zeta^{k}\right) \in E^{1+k}$, and $\left(\alpha_{1}, \ldots, \alpha_{k}\right) \in[0,+\infty)^{k}$.

Definition 1.4. A given finite subset $\mathcal{C}=\left\{W_{k} \mid k \in\{1,2, \ldots, M\}\right\} \subset U$ is said (B, $U$ )saturating if for the following sequence of subspaces of $\mathcal{E}^{j} \subset U$, defined recursively by

$$
\mathcal{E}^{0}:=\operatorname{span} \mathcal{C}, \quad \mathcal{E}^{j+1}:=\mathcal{F}_{\mathrm{B}}\left(\mathcal{E}^{j}\right),
$$

we have that the union $\bigcup_{j \in \mathbb{N}} \mathcal{E}^{j}$ is dense in $H$.

Though, in [28] the author focuses on no-slip boundary conditions, $\left.u\right|_{\partial \Omega}=0$, the results also hold for other boundary conditions. This is also mentioned in [28, Section 2.3. Remark 2.7] where the author considers the case of periodic boundary conditions, and presents an explicit $(\mathrm{B}, \mathrm{D}(A))$-saturating set $\mathcal{C}$ (for the case of $(1,1,1)$-periodic vectors) whose 64 elements are eigenfunctions of the Stokes operator (i.e., the Laplacian). For a general period $q=\left(q_{1}, q_{2}, q_{3}\right) \in\left(\mathbb{R}_{0}\right)^{3}$ the existence of a saturating set is also proven [28, Section 2.3, Theorem 2.5], though the form of the saturating set is less explicit.

Following the proof of the main Theorem 2.2 in [28] we can see that the result holds for a generic setting where we have the subspaces

$$
\mathrm{D}(A) \stackrel{\mathrm{d}, \mathrm{c}}{\longrightarrow} V=\mathrm{D}\left(A^{\frac{1}{2}}\right) \stackrel{\mathrm{d}, \mathrm{c}}{\longrightarrow} H=H^{\prime}, \quad V \subset H \cap H^{1}\left(\Omega, \mathbb{R}^{3}\right), \quad \mathrm{D}(A) \subset H \cap H^{2}\left(\Omega, \mathbb{R}^{3}\right),
$$

with $\mathrm{D}(A)=\{u \in H \mid A u \in H\}$ being the domain Stokes operator $A$ (which depends on the boundary conditions), and where the scalar products

$$
\langle A u, v\rangle_{V^{\prime}, V} \text { and } \quad(A u, A v)_{H}
$$

induce norms in $V$ and $\mathrm{D}(A)$, respectively, which are equivalent to the those inherited from $H^{1}\left(\Omega, \mathbb{R}^{3}\right)$ and $H^{2}\left(\Omega, \mathbb{R}^{3}\right)$, respectively.

Remark 1.3. The notation $S \hookrightarrow R$ above means that the inclusion $S \subseteq R$ is continuous. The letter "d" (resp. "c") means that, in addition, the inclusion is also dense (resp. compact).

Remark 1.4. In the periodic case mentioned above, usually we take a smaller subspace $H_{\text {per }} \subset H$ in order to factor out the kernel of $A$ (as an operator in $H$ ), and guarantee that $(u, v) \mapsto$ $\langle A u, v\rangle_{V_{\mathrm{per}}^{\prime}, V_{\mathrm{per}}}$ defines a scalar product in $V_{\mathrm{per}}:=V \bigcap H_{\mathrm{per}}$. Notice that, for a nonzero constant vector field $u$, and under periodic boundary conditions, we will have $A u=-\nu \Delta u=0$ and thus $\langle A u, u\rangle_{V^{\prime}, V}=0$. Hence, $\langle A u, v\rangle_{V^{\prime}, V}$ does not define a scalar product in $V=H \cap H^{1}\left(\Omega, \mathbb{R}^{3}\right)$.

In particular, the results in 28] hold true for Lions boundary conditions, and we can conclude that approximate controllability for 3D Navier-Stokes equation follows from the existence of a $(\mathrm{B}, \mathrm{D}(A))$-saturating set.

In this paper, we prove that approximate controllability also follows from the existence of a $(\mathrm{L}, \mathrm{D}(A))$-saturating set. Namely, we will prove the following.

Main Theorem. Let $\left(u_{0}, \hat{u}\right) \in V \times V, \varepsilon>0$, and $T>0$. If $\mathcal{C}$ is a $(\mathrm{L}, \mathrm{D}(A))$-saturating set, then we can find a control $\eta \in L^{\infty}\left((0, T), \mathcal{G}^{1}\right)$ so that the solution of system (2) satisfies $|u(T)-\hat{u}|_{V}<\varepsilon$. 
Further, for any given length triplet $L=\left(L_{1}, L_{2}, L_{3}\right)$, we present an explicit (L, D $\left.(A)\right)$ saturating set $\mathcal{C}$ for the $3 \mathrm{D}$ rectangle $\Omega=\left(0, L_{1}\right) \times\left(0, L_{2}\right) \times\left(0, L_{3}\right)$. The elements of $\mathcal{C}$ are 81 eigenfunctions of the Stokes operator, under Lions boundary conditions (cf. Theorem 3.1 hereafter). Though it is not our goal here to find a saturating set with the minimum number of elements as possible, we must say that for some $L$ (maybe, even for all $L$ ) it may exist a saturating set with less elements. In any case, we underline that the existence of a $(\mathrm{L}, \mathrm{D}(A))$-saturating set $\mathcal{C}$ is independent of the viscosity coefficient $\nu$. In particular, the linear space $\mathcal{G}^{1}$, where the control $\eta$ takes its values in, does not change with $\nu$.

Finally, we recall that in [23, 24] an explicit saturating set was found for a 2D rectangle $\Omega=\left(0, L_{1}\right) \times\left(0, L_{2}\right)$ with 8 elements. In [20] a saturating set with 24 elements is presented for the 2D Navier-Stokes system in a Cylinder under Lions boundary conditions i.e., in a channel with Lions boundary conditions in the bounded direction and with periodicity assumption in the unbounded direction).

Remark 1.5. The "L" subcript in Definition 1.2 underlines the fact that the linearization $\mathcal{B}$ of $B$ is used in the recursion step, while in The "B" subcript in Definition 1.4 underlines the fact that the bilinear operator $B$ is used in the recursion step.

1.3. Motivation and further references. An advantage for considering ( $\mathrm{L}, \mathrm{D}(A))$-saturating sets is that the construction of $\mathcal{F}_{\mathrm{L}}(E)$ is easier than the construction of $\mathcal{F}_{\mathrm{B}}(E)$. This is important, when we need to dwell with explicit computations as in the case when we look for explicit saturating sets. Often, the existence of saturating sets is proven by showing that a given explicit set is saturating, which involve essentially explicit computations (Theorem 2.5 in [28] is an exception, but the proof is still strongly based on explicit computations).

For further results concerning the controllability and approximate controllability of NavierStokes (and also other) systems by a control with low finite-dimensional range (independent of the viscosity coefficient) in several domains (including the 2D Sphere and Hemisphere) we refer the reader to [2, 4, 5, 16, 18, 27, 29 31]. We also mention Problem VII raised by A. Agrachev in [1] where the author inquires about the achievable controllability properties for controls taking values in a saturating set whose elements are localized/supported in a small subset $\omega \subset \Omega$. The existence of such saturating sets is an open question (except for 1D Burgers in [20]). The controllability properties implied by such saturating set is an open question. There are some negative results, as for example in the case we consider the $1 \mathrm{D}$ Burgers equations in $\Omega=(0,1)$ and take controls in $L^{2}(\omega, \mathbb{R}), w \subset \Omega$, the approximate controllability fails to hold. Instead, to drive the system from one state $u_{0}=u(0)$ at time $t=0$ to another one $u_{T}=u(T)$ at time $t=T$, we may need $T$ to be big enough. Though we do not consider localized controls here, we refer the reader to the related results in [9, 11,32] and references therein.

Finally we would like to mention that in previous works the existence of a saturating set implied the exact controllability of Galerkin approximations and also the exact controllability onto finite dimensional projections, see for example [3]. To prove these results some geometric control tools are used. We refer also to [7] where the approximate controllability is derived from controllability of Galerkin approximations.

The rest of the paper is organized as follows. In Section 2 we prove that the existence of a $(\mathrm{L}, \mathrm{D}(A))$-saturating set implies the approximate controllability of the Navier-Stokes system. In Section 3 we present a $(\mathrm{L}, \mathrm{D}(A))$-saturating set.

\section{Approximate controllability}

As we said above, in [28] it is proven that the existence of a $(\mathrm{B}, \mathrm{D}(A))$-saturating set implies the approximate controllability of the 3D Navier-Stokes system, at time $T>0$. Here we prove that we can conclude the same controllability property from the existence of a $(\mathrm{L}, \mathrm{D}(A))$-saturating set.

We recall now some definitions from [28]. Hereafter $u_{0} \in V, h \in L_{\text {loc }}^{2}\left(\mathbb{R}_{0}, H\right)$, and $E \subset \mathrm{D}(A)$ is a finite-dimensional subspace. Let us consider the system

$$
\dot{u}+A u+B(u, u)+h=\eta, \quad u(0)=u_{0},
$$


where the control $\eta$ takes its values in $E$.

For simplicity we will denote

$$
I_{T}:=(0, T), \quad \text { and } \quad \overline{I_{T}}:=[0, T], \quad T>0 .
$$

Definition 2.1. Let $T$ be a positive constant. System (5) is said to be $E$-approximately controllable in time $T$ if for any $\varepsilon>0$ and any pair $\left(u_{0}, \hat{u}\right) \in V \times \mathrm{D}(A)$, there exists a control function $\eta \in L^{\infty}\left(I_{T}, E\right)$ and a corresponding solution $u \in C\left(\overline{I_{T}}, V\right) \bigcap L^{2}\left(I_{T}, \mathrm{D}(A)\right)$, such that $|u(T)-\hat{u}|_{V}<\varepsilon$.

Definition 2.2. Let $T, R$, and $\varepsilon$ be positive constants. System (5) is said to be $(\varepsilon, R, E)$ controllable in time $T$ if for any $\left(u_{0}, \hat{u}\right) \in V \times \mathrm{D}(A)$ satisfying $\left|u_{0}\right|_{V} \leq R \geq|\hat{u}|_{\mathrm{D}(A)}$, there exists a control function $\eta \in L^{\infty}\left(I_{T}, E\right)$ and a corresponding solution $u \in C\left(\overline{I_{T}}, V\right) \cap L^{2}\left(I_{T}, \mathrm{D}(A)\right)$ such that $|u(T)-\hat{u}|_{V}<\varepsilon$.

Recall the sequence in Definition 1.4, In [28, Section 2] we find the following results.

Theorem 2.1. If $\mathcal{C}$ is a $(\mathrm{B}, \mathrm{D}(A))$-saturating set, then for any positive $T>0$ the system (5) is $\mathcal{E}^{0}$-approximately controllable at time $T$.

Theorem 2.2. Let $T, R$, and $\varepsilon$ be positive constants. Then system (5) is $(\varepsilon, R, E)$-controllable in time $T$ if it is $\left(\varepsilon, R, \mathcal{F}_{\mathrm{B}}(E)\right)$-controllable at time $T$.

Recall the sequence in Definition 1.2, Here we prove the following.

Theorem 2.3. If $\mathcal{C}$ is a $(\mathrm{L}, \mathrm{D}(A))$-saturating set, then for any positive $T>0$ the system (5) is $\mathcal{G}^{1}$-approximately controllable at time $T$.

Proof. Proceeding as in [28, Section 2.2] we can prove that system (5D) is $\mathcal{G}^{j+1}$-approximately controllable in time $T$, provided $j \in \mathbb{N}$ is big enough. Now for any $u=\sum_{i=1}^{M} u_{i} W_{i} \in \mathcal{G}^{0}$, we have that $B(u, u)=\sum_{i=1}^{M} u_{i} B\left(W_{i}, u\right)$, which implies that $B(u, u) \in \mathcal{F}_{\mathrm{L}}\left(\mathcal{G}^{0}\right)=\mathcal{G}^{1}$, for all $j \geq 0$. Since $\mathcal{G}^{j} \subseteq \mathcal{G}^{j+1}$, for all $j \geq 0$, we have that $\mathcal{G}^{1}+\mathcal{F}_{\mathrm{L}}\left(\mathcal{G}^{j}\right)=\mathcal{G}^{1}+\mathcal{G}^{j+1}=\mathcal{G}^{j+1}$. By the following Theorem 2.4 it follows that system (5) is $\left(\mathcal{G}^{1}+\mathcal{G}^{j}\right)$-approximately controllable in time $T$. Repeating the last argument, we conclude that system (5) is $\left(\mathcal{G}^{1}+\mathcal{G}^{1}\right)$-approximately controllable in time $T$.

Theorem 2.4. Let $T, R$, and $\varepsilon$ be positive constants. Then system (15) is $\left(\varepsilon, R, \mathcal{G}^{1}+E\right)$ controllable in time $T$ if it is $\left(\varepsilon, R, \mathcal{G}^{1}+\mathcal{F}_{\mathrm{L}}(E)\right)$-controllable in time $T$.

Proof. Let us fix $\hat{\varepsilon}>0$ and $\hat{u} \in \mathrm{D}(A)$. Let also $\left(\xi_{0}, \xi_{1}\right) \in L^{\infty}\left(I_{T}, \mathcal{G}^{1}\right) \times L^{\infty}\left(I_{T}, \mathcal{F}_{\mathrm{L}}(E)\right)$ be such that the corresponding solution for

$$
\dot{u}+A u+B(u, u)+h=\xi_{0}+\xi_{1}, \quad u(0)=u_{0},
$$

satisfies

$$
|u(T)-\hat{u}|_{V} \leq \hat{\varepsilon}
$$

We may write, for any $\rho>0$,

$$
\xi_{1}=\eta+\sum_{i=1}^{k} \mathcal{B}\left(a_{i}, b_{i}\right)=\eta+\sum_{i=1}^{k}\left(-B\left(\rho a_{i}-\rho^{-1} b_{i}\right)+\rho^{2} B\left(a_{i}\right)+\rho^{-2} B\left(b_{i}\right)\right)
$$

for suitable $k \in \mathbb{N}_{0}, \eta \in L^{\infty}\left(I_{T}, E\right)$, and suitable pairs $\left(a_{i}, b_{i}\right) \in L^{\infty}\left(I_{T}, \mathcal{C} \times E\right)$. Therefore,

$$
\xi_{1}=\rho^{2} \eta_{a}+\eta_{\rho}+\rho^{-2} \eta_{b}
$$


with

$$
\begin{aligned}
\eta_{a} & :=\sum_{i=1}^{k} B\left(a_{i}, a_{i}\right), \quad \eta_{b}:=\sum_{i=1}^{k} B\left(b_{i}, b_{i}\right) . \\
\eta_{\rho} & :=\eta-\sum_{i=1}^{k} B\left(\rho a_{i}-\rho^{-1} b_{i}, \rho a_{i}-\rho^{-1} b_{i}\right) .
\end{aligned}
$$

Now we rewrite (6) as

$$
\dot{u}+A u+B(u, u)+h=\xi_{0}+\rho^{2} \eta_{a}+\eta_{\rho}+\rho^{-2} \eta_{b}, \quad u(0)=u_{0} .
$$

Since (9) coincides with (6), the solution $u$ of (9) is independent of $\rho$. Let us now consider the solution of the system

$$
\dot{w}_{\rho}+A w_{\rho}+B\left(w_{\rho}, w_{\rho}\right)+h=\xi_{0}+\rho^{2} \eta_{a}+\eta_{\rho}, \quad w_{\rho}(0)=u_{0},
$$

The solution $u$ is known (by Theorem's assumption) to exist for $t \in \overline{I_{T}}$. We show now that the solution $w_{\rho}$ also exists for time $t \in \overline{I_{T}}$, provided $\rho$ is big enough.

Indeed, the difference $z=u-w_{\rho}$ solves

$$
\dot{z}+A z+B(z, z)+\mathcal{B}(u, z)=\rho^{-2} \eta_{b}, \quad z(0)=0,
$$

and we know that $u \in C\left(\overline{I_{T}}, V\right) \subset L^{4}\left(I_{T}, H^{1}\left(\Omega, \mathbb{R}^{3}\right)\right)$ and $\rho^{-2} \eta_{b} \in L^{\infty}\left(I_{T}, H\right) \subset L^{2}\left(I_{T}, H\right)$. Further we know that $\hat{z}=0$ solves system (10) with $\eta_{b}=0$, for time $t \in I_{T}$. Therefore, from [28, Remark 1.9], we can conclude that there exists a unique solution for system (10), for time $t \in I_{T}$, provided $\left|\rho^{-2} \eta_{b}-0\right|_{L^{2}\left(I_{T}, H\right)}$ is small enough. That is, provided $\rho$ is big enough. Furthermore, we have that

$$
\left|u-w_{\rho}\right|_{C\left(\overline{I_{T}}, V\right) \cap L^{2}\left(I_{T}, \mathrm{D}(A)\right)}=|z|_{C\left(\overline{I_{T}}, V\right) \cap L^{2}\left(I_{T}, \mathrm{D}(A)\right)} \leq C \rho^{-2}\left|\eta_{b}\right|_{L^{2}\left(I_{T}, H\right)}
$$

for a suitable constant $C$ depending only on $|u|_{C\left(\overline{I_{T}}, V\right)} \cap L^{2}\left(I_{T}, \mathrm{D}(A)\right)$. See again [28, Remark 1.9]. In particular, for big enough $\rho>0$, we will have

$$
\left|w_{\rho}(T)-u(T)\right|_{V} \leq \hat{\varepsilon} .
$$

Observe that $\eta_{\rho}$ in (88) is in $E-\operatorname{conv}\{B(e, e) \mid e \in E\}$, where conv $S$ stands for the convex cone generated by the subset $S$, that is,

$$
\operatorname{conv} S:=\left\{\sum_{i=1}^{k} \alpha_{i} s_{i} \mid k \in \mathbb{N}, \alpha_{i}>0, s_{i} \in S\right\} .
$$

Hence by Proposition 3.2 in [28] there is $(\tilde{\eta}, \tilde{\zeta}) \in\left(L^{\infty}\left(I_{T}, E\right)\right)^{2}$ so that the corresponding solution for

satisfies

$$
\dot{y}_{\rho}+A\left(y_{\rho}+\tilde{\zeta}\right)+B\left(y_{\rho}+\tilde{\zeta}, y_{\rho}+\tilde{\zeta}\right)+h=\xi_{0}+\rho^{2} \eta_{a}+\tilde{\eta}, \quad y_{\rho}(0)=u_{0},
$$

$$
\left|w_{\rho}-y_{\rho}\right|_{C\left(\overline{I_{T}}, V\right)} \leq \hat{\varepsilon} \text {. }
$$

Remark 2.1. Actually, in [28, Proposition 3.2], it is assumed that $\eta_{\rho} \in \mathcal{F}_{\mathrm{B}}(E)$, but following the proof in [28, Section 3.3], we can see that the the proof is brought to the "imitation" (in short time intervals) of a constant control $\eta_{\rho} \in E-\operatorname{conv}\{B(e, e) \mid e \in E\}$ (see also [28, Section 4.2, proof of Lemma 3.3]).

Now from [28, Proposition 3.1] it follows that there exists a control $\widehat{\eta} \in L^{\infty}\left(I_{T}, E\right)$ such that the solution of the system

$$
\dot{\hat{y}}_{\rho}+A \widehat{y}_{\rho}+B\left(\widehat{y}_{\rho}, \widehat{y}_{\rho}\right)+h=\xi_{0}+\rho^{2} \eta_{a}+\widehat{\eta}, \quad \widehat{w}_{\rho}(0)=u_{0},
$$

satisfies

$$
\left|\widehat{y}_{\rho}(T)-y_{\rho}(T)\right|_{V} \leq \hat{\varepsilon} .
$$

Finally, we observe that $\xi_{0}+\rho^{2} \eta_{a}+\widehat{\eta} \in L^{\infty}\left(I_{T}, \mathcal{G}^{1}+E\right)$, and

$$
\left|\widehat{y}_{\rho}(T)-\hat{u}\right|_{V} \leq 4 \hat{\varepsilon},
$$

which can be concluded from (7), (11), (12), and (13). 


\section{The SATURATing SET}

Here we present a $(\mathrm{L}, \mathrm{D}(A))$-saturating set which consists of a finite number suitable eigenfunctions of the Stokes operator $A$ in the $3 \mathrm{D}$ rectangle

$$
\Omega=\mathrm{R}:=\left(0, L_{1}\right) \times\left(0, L_{2}\right) \times\left(0, L_{3}\right)
$$

under Lions boundary conditions, see (3), where $L_{1}, L_{2}$, and $L_{3}$ are positive real numbers. We follow the arguments in [19, Section 3.5], where the case $L_{1}=L_{2}=L_{3}=\pi$ is considered. Notice that the vector length $L=\left(L_{1}, L_{2}, L_{3}\right)$ plays a role in the explicit computations, and different vector lengths may require slightly different arguments. Recall for example the case of a $2 \mathrm{D}$ rectangle $\left(0, L_{1}\right) \times\left(0, L_{2}\right)$ considered in [25, Section 6.3] where the case of a square $L_{1}=L_{2}$ needs a particular consideration (see also [23]). Recall also the case of the periodic boundary conditions considered in [28, Section 2.3] where in the case $L_{1}=L_{2}=L_{3}$ it is possible to give an explicit form for the $(\mathrm{B}, \mathrm{D}(A))$-saturating set (cf. [28, Remark 2.7], see also [16, Section 4]).

3.1. The saturating set. We will present a saturating set for the rectangle under Lions boundary conditions, which consists of eigenfunctions of $A$.

For a given $k \in \mathbb{N}^{3}$, let $\#_{0}(k)$ stand for the number of vanishing components of $k$. A complete system of eigenfunctions $\left\{Y^{j(k), k}\right\}$ is given by

$$
Y^{j(k), k}:=\left(\begin{array}{l}
w_{1}^{j(k), k} \sin \left(\frac{k_{1} \pi x_{1}}{L_{1}}\right) \cos \left(\frac{k_{2} \pi x_{2}}{L_{2}}\right) \cos \left(\frac{k_{3} \pi x_{3}}{L_{3}}\right) \\
w_{2}^{j(k), k} \cos \left(\frac{k_{1} \pi x_{1}}{L_{1}}\right) \sin \left(\frac{k_{2} \pi x_{2}}{L_{2}}\right) \cos \left(\frac{k_{3} \pi x_{3}}{L_{3}}\right) \\
w_{3}^{j(k), k} \cos \left(\frac{k_{1} \pi x_{1}}{L_{1}}\right) \cos \left(\frac{k_{2} \pi x_{2}}{L_{2}}\right) \sin \left(\frac{k_{3} \pi x_{3}}{L_{3}}\right)
\end{array}\right), \quad \#_{0}(k) \leq 1,
$$

with

$$
\left\{w^{j(k), k} \mid j(k) \in\left\{1,2-\#_{0}(k)\right\}\right\} \subset\{k\}_{0}^{\perp_{[L]}}
$$

a linearly independent and orthogonal family and where

$$
\begin{aligned}
\{k\}_{0}^{\perp_{[L]}} & :=\left\{z \in \mathbb{R}^{3} \backslash\{(0,0,0)\} \mid(z, k)_{[L]}=0, \text { and } z_{i}=0 \text { if } k_{i}=0\right\}, \\
(z, k)_{[L]} & :=\frac{z_{1} k_{1}}{L_{1}}+\frac{z_{2} k_{2}}{L_{2}}+\frac{z_{3} k_{3}}{L_{3}} .
\end{aligned}
$$

Notice that $2-\#_{0}(k)$ is the dimension of the subspace $\{k\}_{0}^{\perp_{[L]}}$ and that the orthogonality of the family $\left\{w^{j(k), k} \mid j(k) \in\left\{1,2-\#_{0}(k)\right\}\right\}$ implies that the family in (14a) is also orthogonal. The completeness of the system in (14a) is shown in [21, Section 6.6].

Example 3.1. The eigenspace associated with a frequency vector $k=(2,4,0)$, is the one spanned by the single eigenfunction $Y^{1, k}$, where we can choose $w^{1, k}=C\left(-4 L_{1}, 2 L_{2}, 0\right)$ for any constant $C \neq 0$. The eigenspace associated with a frequency vector $k=(2,4,5) \in \mathbb{N}_{0}^{3}$, is the one spanned by the eigenfunctions $Y^{1, k}$ and $Y^{2, k}$, where we can choose $\left\{w^{1, k}, w^{2, k}\right\}$ linearly independent in $\operatorname{span}\left\{\left(-4 L_{1}, 2 L_{2}, 0\right),\left(-5 L_{1}, 0,2 L_{3}\right)\right\}$.

Now we are able to present the saturating set in the following Theorem 3.1, whose proof is given in Section 3.5. Before, we need to derive some tools used in the proof.

Theorem 3.1. The set $\mathcal{C}:=\left\{\begin{array}{l|l}Y^{j(n), n} & \begin{array}{l}n \in \mathbb{N}^{3}, \quad 0 \leq n_{i} \leq 3, \\ \#_{0}(n) \leq 1, \quad j(n) \in\left\{1,2-\#_{0}(n)\right\}\end{array}\end{array}\right\}$ is (L, $\left.\mathrm{D}(A)\right)$ saturating.

3.2. The expression for $\left(Y^{k} \cdot \nabla\right) Y^{m}+\left(Y^{m} \cdot \nabla\right) Y^{k}$. Here we will present the expression for the coordinates of $\left(Y^{j(k), k} \cdot \nabla\right) Y^{j(m), m}+\left(Y^{j(m), m} \cdot \nabla\right) Y^{j(k), k}$ for given eigenfunctions as in (14a). In order to shorten the following expressions and simplify the writing, we will write

$$
Y^{k}=Y^{j(k), k}, \quad Y^{m}=Y^{j(m), m}, \quad w^{k}=w^{j(k), k}, \quad \text { and } \quad w^{m}=w^{j(m), m}
$$

by omitting the indexes $j(k), j(m)$. We will also denote

$$
\mathrm{C}_{i}\left(k_{i}\right):=\cos \left(\frac{k_{i} \pi x_{i}}{L_{i}}\right) \quad \text { and } \quad \mathrm{S}_{i}\left(k_{i}\right):=\sin \left(\frac{k_{i} \pi x_{i}}{L_{i}}\right), \quad i \in\{1,2,3\} .
$$


Using these notations, we find

$$
\left(Y^{k} \cdot \nabla\right) Y^{m}=\left(\begin{array}{c}
Y^{k} \cdot w_{1}^{m}\left(\begin{array}{c}
\frac{m_{1} \pi}{L_{1}} \mathrm{C}_{1}\left(m_{1}\right) \mathrm{C}_{2}\left(m_{2}\right) \mathrm{C}_{3}\left(m_{3}\right) \\
-\frac{m_{2} \pi}{L_{2}} \mathrm{~S}_{1}\left(m_{1}\right) \mathrm{S}_{2}\left(m_{2}\right) \mathrm{C}_{3}\left(m_{3}\right) \\
-\frac{m_{3} \pi}{L_{3}} \mathrm{~S}_{1}\left(m_{1}\right) \mathrm{C}_{2}\left(m_{2}\right) \mathrm{S}_{3}\left(m_{3}\right)
\end{array}\right) \\
Y^{k} \cdot w_{2}^{m}\left(\begin{array}{c}
-\frac{m_{1} \pi}{L_{1}} \mathrm{~S}_{1}\left(m_{1}\right) \mathrm{S}_{2}\left(m_{2}\right) \mathrm{C}_{3}\left(m_{3}\right) \\
\frac{m_{2} \pi}{L_{2}} \mathrm{C}_{1}\left(m_{1}\right) \mathrm{C}_{2}\left(m_{2}\right) \mathrm{C}_{3}\left(m_{3}\right) \\
-\frac{m_{3} \pi}{L_{3}} \mathrm{C}_{1}\left(m_{1}\right) \mathrm{S}_{2}\left(m_{2}\right) \mathrm{S}_{3}\left(m_{3}\right)
\end{array}\right) \\
Y^{k} \cdot w_{3}^{m}\left(\begin{array}{c}
-\frac{m_{1} \pi}{L_{1}} \mathrm{~S}_{1}\left(m_{1}\right) \mathrm{C}_{2}\left(m_{2}\right) \mathrm{S}_{3}\left(m_{3}\right) \\
-\frac{m_{2} \pi}{L_{2}} \mathrm{C}_{1}\left(m_{1}\right) \mathrm{S}_{2}\left(m_{2}\right) \mathrm{S}_{3}\left(m_{3}\right) \\
\frac{m_{3} \pi}{L_{3}} \mathrm{C}_{1}\left(m_{1}\right) \mathrm{C}_{2}\left(m_{2}\right) \mathrm{C}_{3}\left(m_{3}\right)
\end{array}\right)
\end{array}\right),
$$

To compute the coordinates of $\left(Y^{k} \cdot \nabla\right) Y^{m}+\left(Y^{m} \cdot \nabla\right) Y^{k}$ it will be useful to define

$$
\beta_{w^{k}, m}^{\star_{1} \star_{2} \star_{3}}:=\frac{\pi}{8}\left(\star_{1} \frac{w_{1}^{k} m_{1}}{L_{1}} \star_{2} \frac{w_{2}^{k} m_{2}}{L_{2}} \star_{3} \frac{w_{3}^{k} m_{3}}{L_{3}}\right), \quad \text { for } \quad\left(\star_{1}, \star_{2}, \star_{3}\right) \in\{+,-\}^{3}
$$

As an illustration, we find the relations $\beta_{w^{k}, m}^{+++}=\frac{\pi}{8}\left(\frac{w_{1}^{k} m_{1}}{L_{1}}+\frac{w_{2}^{k} m_{2}}{L_{2}}+\frac{w_{3}^{k} m_{3}}{L_{3}}\right)$, and $\beta_{w^{m}, k}^{-+-}=$ $\frac{\pi}{8}\left(-\frac{w_{1}^{m} k_{1}}{L_{1}}+\frac{w_{2}^{m} k_{2}}{L_{2}}-\frac{w_{3}^{m} k_{3}}{L_{3}}\right)$.

From straightforward computations we can find

$$
\begin{aligned}
& \left(\left(Y^{k} \cdot \nabla\right) Y^{m}+\left(Y^{m} \cdot \nabla\right) Y^{k}\right)_{1} \\
& =+\left(w_{1}^{m} \beta_{w^{k}, m}^{+++}+w_{1}^{k} \beta_{w^{m}, k}^{+++}\right) \mathrm{S}_{1}\left(k_{1}+m_{1}\right) \mathrm{C}_{2}\left(k_{2}+m_{2}\right) \mathrm{C}_{3}\left(k_{3}+m_{3}\right) \\
& +\left(w_{1}^{m} \beta_{w^{k}, m}^{+++}-w_{1}^{k} \beta_{w^{m}, k}^{+++}\right) \mathrm{S}_{1}\left(k_{1}-m_{1}\right) \mathrm{C}_{2}\left(k_{2}-m_{2}\right) \mathrm{C}_{3}\left(k_{3}-m_{3}\right) \\
& +\left(w_{1}^{m} \beta_{w^{k}, m}^{++-}+w_{1}^{k} \beta_{w^{m}, k}^{++-}\right) \mathrm{S}_{1}\left(k_{1}+m_{1}\right) \mathrm{C}_{2}\left(k_{2}+m_{2}\right) \mathrm{C}_{3}\left(k_{3}-m_{3}\right) \\
& +\left(w_{1}^{m} \beta_{w^{k}, m}^{++-}-w_{1}^{k} \beta_{w^{m}, k}^{++-}\right) \mathrm{S}_{1}\left(k_{1}-m_{1}\right) \mathrm{C}_{2}\left(k_{2}-m_{2}\right) \mathrm{C}_{3}\left(k_{3}+m_{3}\right) \\
& +\left(w_{1}^{m} \beta_{w^{k}, m}^{+-+}+w_{1}^{k} \beta_{w^{m}, k}^{+-+}\right) \mathrm{S}_{1}\left(k_{1}+m_{1}\right) \mathrm{C}_{2}\left(k_{2}-m_{2}\right) \mathrm{C}_{3}\left(k_{3}+m_{3}\right) \\
& +\left(w_{1}^{m} \beta_{w^{k}, m}^{+-+}-w_{1}^{k} \beta_{w^{m}, k}^{+-+}\right) \mathrm{S}_{1}\left(k_{1}-m_{1}\right) \mathrm{C}_{2}\left(k_{2}+m_{2}\right) \mathrm{C}_{3}\left(k_{3}-m_{3}\right) \\
& +\left(w_{1}^{m} \beta_{w^{k}, m}^{+--}+w_{1}^{k} \beta_{w^{m}, k}^{+--}\right) \mathrm{S}_{1}\left(k_{1}+m_{1}\right) \mathrm{C}_{2}\left(k_{2}-m_{2}\right) \mathrm{C}_{3}\left(k_{3}-m_{3}\right) \\
& +\left(w_{1}^{m} \beta_{w^{k}, m}^{+--}-w_{1}^{k} \beta_{w^{m}, k}^{+--}\right) \mathrm{S}_{1}\left(k_{1}-m_{1}\right) \mathrm{C}_{2}\left(k_{2}+m_{2}\right) \mathrm{C}_{3}\left(k_{3}+m_{3}\right), \\
& \left(\left(Y^{k} \cdot \nabla\right) Y^{m}+\left(Y^{m} \cdot \nabla\right) Y^{k}\right)_{2} \\
& =+\left(w_{2}^{m} \beta_{w^{k}, m}^{+++}+w_{2}^{k} \beta_{w^{m}, k}^{+++}\right) \mathrm{C}_{1}\left(k_{1}+m_{1}\right) \mathrm{S}_{2}\left(k_{2}+m_{2}\right) \mathrm{C}_{3}\left(k_{3}+m_{3}\right) \\
& +\left(w_{2}^{m} \beta_{w^{k}, m}^{+++}-w_{2}^{k} \beta_{w^{m}, k}^{+++}\right) \mathrm{C}_{1}\left(k_{1}-m_{1}\right) \mathrm{S}_{2}\left(k_{2}-m_{2}\right) \mathrm{C}_{3}\left(k_{3}-m_{3}\right) \\
& +\left(w_{2}^{m} \beta_{w^{k}, m}^{++-}+w_{2}^{k} \beta_{w^{m}, k}^{++-}\right) \mathrm{C}_{1}\left(k_{1}+m_{1}\right) \mathrm{S}_{2}\left(k_{2}+m_{2}\right) \mathrm{C}_{3}\left(k_{3}-m_{3}\right) \\
& +\left(w_{2}^{m} \beta_{w^{k}, m}^{++-}-w_{2}^{k} \beta_{w^{m}, k}^{++-}\right) \mathrm{C}_{1}\left(k_{1}-m_{1}\right) \mathrm{S}_{2}\left(k_{2}-m_{2}\right) \mathrm{C}_{3}\left(k_{3}+m_{3}\right) \\
& +\left(-w_{2}^{m} \beta_{w^{k}, m}^{+-+}+w_{2}^{k} \beta_{w^{m}, k}^{+-+}\right) \mathrm{C}_{1}\left(k_{1}+m_{1}\right) \mathrm{S}_{2}\left(k_{2}-m_{2}\right) \mathrm{C}_{3}\left(k_{3}+m_{3}\right) \\
& +\left(-w_{2}^{m} \beta_{w^{k}, m}^{+-+}-w_{2}^{k} \beta_{w^{m}, k}^{+-+}\right) \mathrm{C}_{1}\left(k_{1}-m_{1}\right) \mathrm{S}_{2}\left(k_{2}+m_{2}\right) \mathrm{C}_{3}\left(k_{3}-m_{3}\right) \\
& +\left(-w_{2}^{m} \beta_{w^{k}, m}^{+--}+w_{2}^{k} \beta_{w^{m}, k}^{+--}\right) \mathrm{C}_{1}\left(k_{1}+m_{1}\right) \mathrm{S}_{2}\left(k_{2}-m_{2}\right) \mathrm{C}_{3}\left(k_{3}-m_{3}\right) \\
& +\left(-w_{2}^{m} \beta_{w^{k}, m}^{+--}-w_{2}^{k} \beta_{w^{m}, k}^{+--}\right) \mathrm{C}_{1}\left(k_{1}-m_{1}\right) \mathrm{S}_{2}\left(k_{2}+m_{2}\right) \mathrm{C}_{3}\left(k_{3}+m_{3}\right),
\end{aligned}
$$




$$
\begin{aligned}
\left(\left(Y^{k} \cdot \nabla\right)\right. & \left.Y^{m}+\left(Y^{m} \cdot \nabla\right) Y^{k}\right)_{3} \\
= & +\left(w_{3}^{m} \beta_{w^{k}, m}^{+++}+w_{3}^{k} \beta_{w^{m}, k}^{+++}\right) \mathrm{C}_{1}\left(k_{1}+m_{1}\right) \mathrm{C}_{2}\left(k_{2}+m_{2}\right) \mathrm{S}_{3}\left(k_{3}+m_{3}\right) \\
& +\left(w_{3}^{m} \beta_{w^{k}, m}^{+++}-w_{3}^{k} \beta_{w^{m}, k}^{+++}\right) \mathrm{C}_{1}\left(k_{1}-m_{1}\right) \mathrm{C}_{2}\left(k_{2}-m_{2}\right) \mathrm{S}_{3}\left(k_{3}-m_{3}\right) \\
& +\left(-w_{3}^{m} \beta_{w^{k}, m}^{++-}+w_{3}^{k} \beta_{w^{m}, k}^{++-}\right) \mathrm{C}_{1}\left(k_{1}+m_{1}\right) \mathrm{C}_{2}\left(k_{2}+m_{2}\right) \mathrm{S}_{3}\left(k_{3}-m_{3}\right) \\
& +\left(-w_{3}^{m} \beta_{w^{k}, m}^{++-}-w_{3}^{k} \beta_{w^{m}, k}^{++-}\right) \mathrm{C}_{1}\left(k_{1}-m_{1}\right) \mathrm{C}_{2}\left(k_{2}-m_{2}\right) \mathrm{S}_{3}\left(k_{3}+m_{3}\right) \\
& +\left(w_{3}^{m} \beta_{w^{k}, m}^{+-+}+w_{3}^{k} \beta_{w^{m}, k}^{+-+}\right) \mathrm{C}_{1}\left(k_{1}+m_{1}\right) \mathrm{C}_{2}\left(k_{2}-m_{2}\right) \mathrm{S}_{3}\left(k_{3}+m_{3}\right) \\
& +\left(w_{3}^{m} \beta_{w^{k}, m}^{+-+}-w_{3}^{k} \beta_{w^{m}, k}^{+-+}\right) \mathrm{C}_{1}\left(k_{1}-m_{1}\right) \mathrm{C}_{2}\left(k_{2}+m_{2}\right) \mathrm{S}_{3}\left(k_{3}-m_{3}\right) \\
& +\left(-w_{3}^{m} \beta_{w^{k}, m}^{+--}+w_{3}^{k} \beta_{w^{m}, k}^{+--}\right) \mathrm{C}_{1}\left(k_{1}+m_{1}\right) \mathrm{C}_{2}\left(k_{2}-m_{2}\right) \mathrm{S}_{3}\left(k_{3}-m_{3}\right) \\
& +\left(-w_{3}^{m} \beta_{w^{k}, m}^{+--}-w_{3}^{k} \beta_{w^{m}, k}^{+--}\right) \mathrm{C}_{1}\left(k_{1}-m_{1}\right) \mathrm{C}_{2}\left(k_{2}+m_{2}\right) \mathrm{S}_{3}\left(k_{3}+m_{3}\right) .
\end{aligned}
$$

Accordingly to Definition 1.2 , we would need to compute the orthogonal projection $\mathcal{B}\left(Y^{k}, Y^{m}\right)=$ $\Pi\left(\left(Y^{k} \cdot \nabla\right) Y^{m}+\left(Y^{m} \cdot \nabla\right) Y^{k}\right)$, onto $H$. However, we will manage to use only the coordinates in (16) instead of the explicit expression for $\mathcal{B}\left(Y^{k}, Y^{m}\right)$ (cf. Section 3.4). The expression for $\mathcal{B}\left(Y^{k}, Y^{m}\right)$ can be more cumbersome than the expressions in (16). For the case $L=$ $\left(L_{1}, L_{2}, L_{3}\right)=(\pi, \pi, \pi)$, the explicit expression for $\mathcal{B}\left(Y^{k}, Y^{m}\right)$ can be found in [19, Section 3.5.1].

3.3. A difference between $2 \mathrm{D}$ and $3 \mathrm{D}$ cases. For the case of $2 \mathrm{D}$ Navier-Stokes equation on a rectangle under Lions boundary conditions, treated in [23], it holds that $B\left(W^{n}, W^{n}\right)=0$ for an eigenfunction $W^{n}$ of the corresponding 2D Stokes operator (cf. [25, Section 4.5]). This can be seen from the fact that vectors fields in $u \in H$ can be identified with a so-called stream function $\phi_{u}$, as $u=\nabla^{\perp} \phi_{u}$, and that we have the vorticity relations $\nabla^{\perp} \cdot u=-\Delta \phi_{u}$ and $\nabla^{\perp} \cdot B(u, u)=-u \cdot \nabla\left(\nabla^{\perp} \cdot u\right)=\nabla \phi_{u} \cdot \nabla^{\perp}\left(\nabla^{\perp} \cdot u\right)=-\nabla \phi_{u} \cdot \Delta u$. Thus $\nabla^{\perp} \cdot B\left(W^{n}, W^{n}\right)=\lambda_{n} \nabla \phi_{W^{n}}$. $W^{n}=\lambda_{n} \nabla \phi_{W^{n}} \cdot \nabla^{\perp} \phi_{W^{n}}=0$, where $\lambda_{n}$ is the eigenvalue associated to $W^{n}, \Pi(-\Delta) W_{n}=\lambda_{n} W_{n}$.

From Theorem 3.2 below, in the case of the 3D rectangle, the identity $B\left(Y^{k}, Y^{k}\right)=0$ does not hold for all eigenfunctions $Y^{k}$ (cf. the case of the 1D Burgers equation studied in [20]).

Theorem 3.2. For an eigenfuntion $Y^{k}=Y^{j(k), k}$ as in (14), we have

$$
\begin{array}{ll}
B\left(Y^{k}, Y^{k}\right) \neq 0, & \text { if } \quad \#_{0}(k)=0, \\
B\left(Y^{k}, Y^{k}\right)=0, & \text { if } \quad \#_{0}(k)=1 .
\end{array}
$$

Proof. Indeed in the case $\#_{0}(k)=0$, since $0=\left(w_{1}^{k}, k\right)_{[L]}=\frac{w_{1}^{k} k_{1}}{L_{1}}+\frac{w_{2}^{k} k_{2}}{L_{2}}+\frac{w_{3}^{k} k_{3}}{L_{3}}$, from (16) with $m=k$, we can rewrite the first coordinate in short form as follows

$$
\begin{aligned}
& \left(\left(Y^{k} \cdot \nabla\right) Y^{k}\right)_{1} \\
= & -\frac{\pi}{4} w_{1}^{k} \frac{w_{3}^{k} k_{3}}{L_{3}} \mathrm{~S}_{1}\left(2 k_{1}\right) \mathrm{C}_{2}\left(2 k_{2}\right)-\frac{\pi}{4} w_{1}^{k} \frac{w_{2}^{k} k_{2}}{L_{2}} \mathrm{~S}_{1}\left(2 k_{1}\right) \mathrm{C}_{3}\left(2 k_{3}\right)+\frac{\pi}{4} w_{1}^{k} \frac{w_{1}^{k} k_{1}}{L_{1}} \mathrm{~S}_{1}\left(2 k_{1}\right) \\
= & -\frac{\pi}{2} w_{1}^{k} \sin \left(\frac{2 k_{1} \pi x_{1}}{L_{1}}\right)\left(\frac{w_{3}^{k} k_{3}}{L_{3}} \cos ^{2}\left(\frac{k_{2} \pi x_{2}}{L_{2}}\right)+\frac{w_{2}^{k} k_{2}}{L_{2}} \cos ^{2}\left(\frac{k_{3} \pi x_{3}}{L_{3}}\right)\right) .
\end{aligned}
$$

Proceeding analogously for the other two coordinates, we obtain

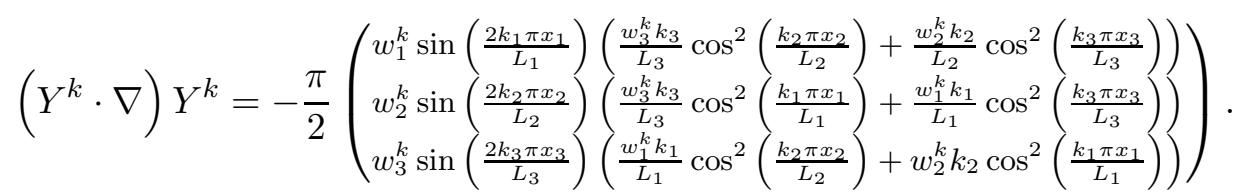

Assuming that $B\left(Y^{k}, Y^{k}\right)=\Pi\left(\left(Y^{k} \cdot \nabla\right) Y^{k}\right)=0$, there would exist a function $g$ such that $\left(Y^{k} \cdot \nabla\right) Y^{k}=\nabla g$ because $H^{\perp}=\left\{\nabla g \mid g \in H^{1}(\Omega, \mathbb{R})\right\}$ (cf. [33, Section 2.5]), which implies 
that $\operatorname{curl}\left(\left(Y^{k} \cdot \nabla\right) Y^{k}\right)=\operatorname{curl}(\nabla g)=0$. That is,

$$
0=\operatorname{curl}\left(\left(Y^{k} \cdot \nabla\right) Y^{k}\right)=\frac{\pi^{2}}{2}\left(\begin{array}{l}
\frac{w_{1}^{k} k_{1}}{L_{1}} \sin \left(\frac{2 k_{2} \pi x_{2}}{L_{2}}\right) \sin \left(\frac{2 k_{3} \pi x_{3}}{L_{3}}\right)\left(\frac{w_{3}^{k} k_{2}}{L_{2}}-\frac{w_{2}^{k} k_{3}}{L_{3}}\right) \\
\frac{w_{2}^{k} k_{2}}{L_{2}} \sin \left(\frac{2 k_{3} x_{3}}{L_{3}}\right) \sin \left(\frac{2 k_{1} \pi x_{1}}{L_{1}}\right)\left(\frac{w_{1}^{k} k_{3}}{L_{3}}-\frac{w_{3}^{k} k_{1}}{L_{1}}\right) \\
\frac{w_{3}^{k} k_{3}}{L_{3}} \sin \left(\frac{2 k_{1} x_{1}}{L_{1}}\right) \sin \left(\frac{2 k_{2} x_{2}}{L_{2}}\right)\left(\frac{w_{2}^{k} k_{1}}{L_{1}}-\frac{w_{1}^{k} k_{2}}{L_{2}}\right)
\end{array}\right) .
$$

We will prove that this equality cannot hold if $\#_{0}(k)=0$. We start by proving that, in this case, no component of $w^{k}$ is vanishing. Indeed, if for example $w_{1}^{k}=0$, we would have $\frac{w_{2}^{k} k_{2}}{L_{2}}=-\frac{w_{3}^{k} k_{3}}{L_{3}}$. Then, (18) would give us

$$
\begin{aligned}
0 & =\left(\begin{array}{c}
0 \\
-\frac{w_{2}^{k} k_{2}}{L_{2}} \frac{w_{3}^{k} k_{1}}{L_{1}} \sin \left(\frac{2 k_{3} \pi x_{3}}{L_{3}}\right) \sin \left(\frac{2 k_{1} \pi x_{1}}{L_{1}}\right) \\
\frac{w_{3}^{k} k_{3}}{L_{3}} \frac{w_{2}^{k} k_{1}}{L_{1}} \sin \left(\frac{2 k_{1} \pi x_{1}}{L_{1}}\right) \sin \left(\frac{2 k_{2} \pi x_{2}}{L_{2}}\right)
\end{array}\right) \\
& =\frac{w_{3}^{k} k_{3}}{L_{3}} \frac{k_{1}}{L_{1}} \sin \left(\frac{2 k_{1} \pi x_{1}}{L_{1}}\right)\left(\begin{array}{c}
0 \\
w_{3}^{k} \sin \left(\frac{2 k_{3} \pi x_{3}}{L_{3}}\right) \\
w_{2}^{k} \sin \left(\frac{2 k_{2} \pi x_{2}}{L_{2}}\right)
\end{array}\right) .
\end{aligned}
$$

Since $k \in \mathbb{N}_{0}^{3}$, it follows that necessarily $\left(w_{3}^{k}\right)^{2}=w_{2}^{k} w_{3}^{k}=0$, which in turn leads us to $w^{k}=$ $(0,0,0)$. This contradicts the fact that by the definition $w^{k} \neq 0$, because the family $\left\{w^{j(k), k} \mid\right.$ $\left.j(k) \in\left\{1,2-\#_{0}(k)\right\}\right\}$ must be linearly independent. Thus $w_{1}^{k} \neq 0$. A similar argument leads us to $w_{2}^{k} \neq 0$ and $w_{3}^{k} \neq 0$.

Now, since all components of $w^{k}$ are different from 0 , from (18), we have

$$
\frac{w_{3}^{k} k_{2}}{L_{2}}-\frac{w_{2}^{k} k_{3}}{L_{3}}=\frac{w_{1}^{k} k_{3}}{L_{3}}-\frac{w_{3}^{k} k_{1}}{L_{1}}=\frac{w_{2}^{k} k_{1}}{L_{1}}-\frac{w_{1}^{k} k_{2}}{L_{2}}=0
$$

that is $k^{L} \times w^{k}=0$, with $k^{L}:=\left(\frac{k_{1}}{L_{1}}, \frac{k_{2}}{L_{2}}, \frac{k_{3}}{L_{3}}\right)$. Furthermore $w^{k} \cdot k^{L}=\left(w^{k}, k\right)_{[L]}=0$ and from the triple vector product relation

$$
k^{L} \times\left(k^{L} \times w^{k}\right)=\left(k^{L} \cdot w^{k}\right) k^{L}-\left(k^{L} \cdot k^{L}\right) w^{k},
$$

(cf. [6, Section 2.35]) it follows that $0=0-\left(k^{L} \cdot k^{L}\right) w^{k}=-\left|k^{L}\right|^{2} w^{k}$ which leads to the contradiction $w^{k}=0$. Therefore we can conclude that $B\left(Y^{k}, Y^{k}\right) \neq 0$ for all $k \in \mathbb{N}_{0}^{3}$.

In the case $\#_{0}(k)=1$, for example if $k_{3}=0$, then $w_{3}^{k}=0$ and from (17) we obtain

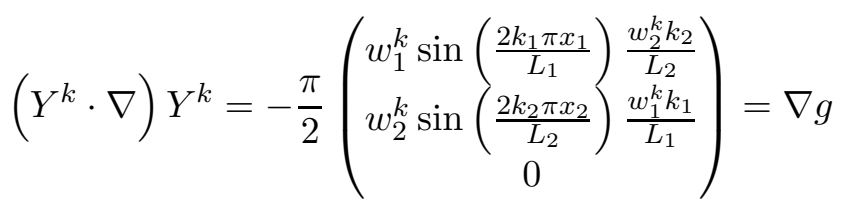

with $g=\frac{w_{1}^{k} w_{2}^{k}}{4}\left(\frac{k_{2} L_{1}}{k_{1} L_{2}} \cos \left(\frac{2 k_{1} \pi x_{1}}{L_{1}}\right)+\frac{k_{1} L_{2}}{k_{2} L_{1}} \cos \left(\frac{2 k_{2} \pi x_{2}}{L_{2}}\right)\right)$. Thus $B\left(Y^{k}, Y^{k}\right)=0$, if $k_{3}=0$. A similar argument gives us that $B\left(Y^{k}, Y^{k}\right)=0$ when $k_{i}=0$, for $i \in\{1,2\}$.

3.4. Avoiding the computation of $\mathcal{B}\left(Y^{k}, Y^{m}\right)$. We present here an auxiliary result which will allow us to work with the coordinates in (16), avoiding to derive (and avoiding the need to work with) the explicit expression for the projection $\mathcal{B}\left(Y^{k}, Y^{m}\right)=\Pi\left(\left\langle Y^{k} \cdot \nabla\right\rangle Y^{m}+\left\langle Y^{m} \cdot \nabla\right\rangle Y^{k}\right)$ (cf. Definition 1.2).

With $k \in \mathbb{N}^{3}$, let us define the functions

$$
\begin{aligned}
& \psi_{1}^{k}=\psi_{1}^{k}(x)=\sin \left(\frac{k_{1} \pi x_{1}}{L_{1}}\right) \cos \left(\frac{k_{2} \pi x_{2}}{L_{2}}\right) \cos \left(\frac{k_{3} \pi x_{3}}{L_{3}}\right), \\
& \psi_{2}^{k}=\psi_{2}^{k}(x)=\cos \left(\frac{k_{1} \pi x_{1}}{L_{1}}\right) \sin \left(\frac{k_{2} \pi x_{2}}{L_{2}}\right) \cos \left(\frac{k_{3} \pi x_{3}}{L_{3}}\right), \\
& \psi_{3}^{k}=\psi_{3}^{k}(x)=\cos \left(\frac{k_{1} \pi x_{1}}{L_{1}}\right) \cos \left(\frac{k_{2} \pi x_{2}}{L_{2}}\right) \sin \left(\frac{k_{3} \pi x_{3}}{L_{3}}\right),
\end{aligned}
$$


and the vector functions

$$
\mathcal{Y}_{z}^{k}=\left(\begin{array}{c}
z_{1} \psi_{1}^{k} \\
z_{2} \psi_{2}^{k} \\
z_{3} \psi_{3}^{k}
\end{array}\right), \quad k \in \mathbb{N}^{3}, z \in \mathbb{R}^{3} .
$$

we observe that for the eigenfunctions $Y^{k}=Y^{j(k), k}$ in (14a), we have

$$
Y^{j(k), k}=\mathcal{Y}_{w^{j(k) . k}}^{k} \text { with } k \in \mathbb{N}^{3}, \#_{0}(k) \leq 1, j(k) \in\left\{1,2-\#_{0}(k)\right\} .
$$

Observe also that if $m \neq k$ then $\left(\mathcal{Y}_{z}^{k}, \mathcal{Y}_{w}^{m}\right)_{L^{2}\left(\Omega, \mathbb{R}^{3}\right)}=0$ for all $z, w \in \mathbb{R}^{3}$, because we have $\left(\psi_{i}^{k}, \psi_{i}^{m}\right)_{L^{2}\left(\left(0, L_{i}\right), R\right)}=0$ for all $i \in\{1,2,3\}$. From (16), we observe that

$$
\left(Y^{k} \cdot \nabla\right) Y^{m}+\left(Y^{m} \cdot \nabla\right) Y^{k}=\sum_{\substack{n=\left(k\left(\star_{1} \star_{2} \star_{3}\right) m\right)^{+} \\\left(\star_{1}, \star_{2}, \star_{3}\right) \in\{-,+\}^{3}}} \mathcal{Y}_{z^{n}}^{n},
$$

where

$$
\left(k\left(\star_{1} \star_{2} \star_{3}\right) m\right)^{+}:=\left(\left|k_{1} \star_{1} m_{1}\right|,\left|k_{2} \star_{2} m_{2}\right|,\left|k_{3} \star_{3} m_{3}\right|\right),
$$

and for suitable vectors $z^{n}=\left(z_{1}^{n}, z_{2}^{n}, z_{3}^{n}\right) \in \mathbb{R}^{3}$ (depending on the parameters $k, m, w^{m}$ and $\left.w^{k}\right)$. Thus the projection

$$
\mathcal{B}\left(Y^{m}, Y^{k}\right)=\sum_{\substack{n=\left(k\left(\star_{1} \star_{2} \star_{3}\right) m\right)^{+} \\\left(\star_{1}, \star_{2}, \star_{3}\right) \in\{-,+\}^{3} \\ \# 0(n) \leq 1, j(n) \in\left\{1,2-\#_{0}(n)\right\}}} \alpha^{j(n), n} Y^{j(n), n}
$$

satisfies, for any $n$,

$$
\sum_{j(n) \in\left\{1,2-\#_{0}(n)\right\}} \alpha^{j(n), n} Y^{j(n), n}=\Pi \mathcal{Y}_{z^{n}}^{n}=\Pi\left(\begin{array}{c}
z_{1}^{n} \psi_{1}^{n} \\
z_{2}^{n} \psi_{2}^{n} \\
z_{3}^{n} \psi_{3}^{n}
\end{array}\right) .
$$

Lemma 3.3. Let us be given $\alpha, \gamma \in \mathbb{R}^{3}$ and $k \in \mathbb{N}_{0}^{3}$. Then the family $\{\alpha, \gamma, k\}$ is linearly independent if, and only if, the family $\left\{\Pi \mathcal{Y}_{\alpha}^{k}, \Pi \mathcal{Y}_{\gamma}^{k}\right\}$ is linearly independent. In either case

$$
\operatorname{span}\left\{\Pi \mathcal{Y}_{\alpha}^{k}, \Pi \mathcal{Y}_{\gamma}^{k}\right\}=\operatorname{span} Y^{\{1,2\}, k} .
$$

Proof. Let us fix a basis $\left\{w^{1, k}, w^{2, k}\right\}$ for $\{k\}_{0}^{\perp}=\{k\}^{\perp}$.

Given $\alpha, \gamma \in \mathbb{R}^{3}$, since $\left\{w^{1, k}, w^{2, k}, k\right\}$ is a basis in $\mathbb{R}^{3}$, we can write (in an unique way)

$$
\begin{aligned}
& \alpha=\alpha^{1, k} w^{1, k}+\alpha^{2, k} w^{2, k}+\alpha_{0} k, \\
& \gamma=\gamma^{1, k} w^{1, k}+\gamma^{2, k} w^{2, k}+\gamma_{0} k .
\end{aligned}
$$

and it follows that

$$
\begin{aligned}
& \mathcal{Y}_{\alpha}^{k}=\alpha^{1, k} Y^{1, k}+\alpha^{2, k} Y^{2, k}+\alpha_{0} \mathcal{Y}_{k}^{k}, \\
& \mathcal{Y}_{\gamma}^{k}=\gamma^{1, k} Y^{1, k}+\gamma^{2, k} Y^{2, k}+\gamma_{0} \mathcal{Y}_{k}^{k} .
\end{aligned}
$$

Since $\mathcal{Y}_{k}^{k}=\nabla\left(-\cos \left(\frac{k_{1} \pi x_{1}}{L_{1}}\right) \cos \left(\frac{k_{2} \pi x_{2}}{L_{2}}\right) \cos \left(\frac{k_{3} \pi x_{3}}{L_{3}}\right)\right)$, we obtain

$$
\begin{aligned}
\Pi \mathcal{Y}_{\alpha}^{k} & =\alpha^{1, k} Y^{1, k}+\alpha^{2, k} Y^{2, k}, \\
\Pi \mathcal{Y}_{\gamma}^{k} & =\gamma^{1, k} Y^{1, k}+\gamma^{2, k} Y^{2, k} .
\end{aligned}
$$

Now, it is clear that $\operatorname{span}\left\{\Pi \mathcal{Y}_{\alpha}^{k}, \Pi \mathcal{Y}_{\gamma}^{k}\right\}=\operatorname{span} Y^{\{1,2\}, k}$ if, and only if, the family $\left\{\Pi \mathcal{Y}_{\alpha}^{k}, \Pi \mathcal{Y}_{\gamma}^{k}\right\}$ is linearly independent. Recall that $\left\{Y^{1, k}, Y^{2, k}\right\}$ is linearly independent by definition. 
Observe that given $(r, s) \in \mathbb{R}^{2}$ such that $r \Pi \mathcal{Y}_{\alpha}^{k}+s \Pi \mathcal{Y}_{\gamma}^{k}=0$, we have (using (23)) that $\left(r \alpha^{1, k}+s \gamma^{1, k}\right) Y^{1, k}+\left(r \alpha^{2, k}+s \gamma^{2, k}\right) Y^{2, k}=0$ and, since $\left\{Y^{1, k}, Y^{2, k}\right\}$ is linearly independent, we find that $\left(\begin{array}{cc}\alpha^{1, k} & \gamma^{1, k} \\ \alpha^{2, k} & \gamma^{2, k}\end{array}\right)\left(\begin{array}{l}r \\ s\end{array}\right)=\left(\begin{array}{l}0 \\ 0\end{array}\right)$. Therefore

$\left\{\Pi \mathcal{Y}_{\alpha}^{k}, \Pi \mathcal{Y}_{\gamma}^{k}\right\}$ is linearly independent if, and only if, $\operatorname{det}\left(\begin{array}{ll}\alpha^{1, k} & \alpha^{2, k} \\ \gamma^{1, k} & \gamma^{2, k}\end{array}\right) \neq 0$.

Since $\left\{w^{1, k}, w^{2, k}, k\right\}$ is linearly independent, a similar argument (using (22) together with $k=0 w^{1, k}+0 w^{2, k}+1 k$ ) leads us to

$$
\{\alpha, \gamma, k\} \text { is linearly independent if, and only if, } \operatorname{det}\left(\begin{array}{ccc}
\alpha^{1, k} & \alpha^{2, k} & \alpha_{0} \\
\gamma^{1, k} & \gamma^{2, k} & \gamma_{0} \\
0 & 0 & 1
\end{array}\right) \neq 0 .
$$

The Lemma follows from (24) and (25).

3.5. Proof of Theorem 3.1, Introducing the family of sets

$$
\begin{aligned}
& \mathcal{S}^{q}:=\left\{n \in \mathbb{N}^{3} \mid 0 \leq n_{i} \leq q, \#_{0}(n) \leq 1\right\}, \\
& \mathcal{C}^{q}:=\left\{Y^{j(n), n} \mid n \in \mathcal{S}^{q}, j(n) \in\left\{1,2-\#_{0}(n)\right\}\right\}, \quad q \in \mathbb{N}, \quad q \geq 3,
\end{aligned}
$$

and recalling the sequence in Definition 1.2, we can see that Theorem 3.1 is a corollary of the following inclusions

$$
\mathcal{C}^{3} \subseteq \mathcal{G}^{0}, \quad \text { and } \quad \mathcal{C}^{q} \subseteq \mathcal{G}^{q-1}, \quad \text { for all } \quad q \in \mathbb{N}, \quad q \geq 3,
$$

which we will prove by induction.

Base step. By definition, $\mathcal{C}=\mathcal{C}^{3}$ and $\operatorname{span} \mathcal{C}=\mathcal{G}^{0} \subseteq \mathcal{G}^{2}$. Therefore

$$
\text { Inclusions (27) holds for } q=3 \text {. }
$$

Induction step. The induction hypothesis is

$\mathcal{C}^{3} \subseteq \mathcal{G}^{0}$ and the inclusion $\mathcal{C}^{q} \subseteq \mathcal{G}^{q-1}$ holds true for a given $q \in \mathbb{N}, q \geq 3$.

(IH.R-eq.29)

We want to prove that $\mathcal{C}^{q+1} \subseteq \mathcal{G}^{q}$.

Notice that

$$
\left.\mathcal{C}^{q+1}:=\left\{Y^{1, n} \mid n \in \mathcal{S}^{q+1}, \#_{0}(n)=1\right\} \bigcup\left\{Y^{1, n}, Y^{2, n} \mid n \in \mathcal{S}^{q+1}, \#_{0}(n)=0\right\}\right\},
$$

We will consider the cases $\#_{0}(n)=1$ and $\#_{0}(n)=0$ separately.

- The case $n \in \mathcal{C}^{q+1}$ and $\#_{0}(n)=1$. Suppose that $k \in \mathbb{N}^{3}, \#_{0}(k)=1$, and $k_{3}=0$. We can see that, up to a constant $C \neq 0, Y^{k}=C\left(\begin{array}{c}W_{\bar{k}} \\ 0\end{array}\right)$, where for simplicity we denoted $Y^{k}=Y^{1, k}$ and $W_{\bar{k}}:=\left(\begin{array}{c}\frac{-k_{2} \pi}{L_{2}} \mathrm{~S}_{1}\left(k_{1}\right) \mathrm{C}_{2}\left(k_{2}\right) \\ \frac{k_{1} \pi}{L_{1}} \mathrm{C}_{1}\left(k_{1}\right) \mathrm{S}_{2}\left(k_{2}\right)\end{array}\right)$, with $\bar{k}:=\left(k_{1}, k_{2}\right)$. Notice that $W_{\bar{k}}$ is an eigenfunction of the Stokes operator in the $2 \mathrm{D}$ rectangle $\mathrm{R}_{2}=\left(0, L_{1}\right) \times\left(0, L_{2}\right)$, as observed in [23, Section 2.2]. Now let also $m \in \mathbb{N}^{3}, \#_{0}(m)=1$, and $m_{3}=0$. Then, we can see that

$$
\left(\left(Y^{k} \cdot \nabla\right) Y^{m}+\left(Y^{m} \cdot \nabla\right) Y^{k}\right)=\left(\begin{array}{c}
\left(\left(W_{\bar{k}} \cdot \nabla_{2}\right) W_{\bar{m}}+\left(W_{\bar{m}} \cdot \nabla_{2}\right) W_{\bar{k}}\right) \\
0
\end{array}\right)
$$

where $\nabla_{2}$ is the gradient on the rectangle $\mathrm{R}_{2}$, that is, on the variables $\left(x_{1}, x_{2}\right)$.

Now, on one hand we can write

$$
\left(\left(Y^{k} \cdot \nabla\right) Y^{m}+\left(Y^{m} \cdot \nabla\right) Y^{k}\right)=\mathcal{B}\left(Y^{k}, Y^{m}\right)+\nabla q
$$

where $\mathcal{B}\left(Y^{k}, Y^{m}\right) \in H$ and $q \in H^{1}\left(\mathrm{R}, \mathbb{R}^{3}\right)$. On the other hand we can write

$$
\left(W_{\bar{k}} \cdot \nabla_{2}\right) W_{\bar{m}}+\left(W_{\bar{m}} \cdot \nabla_{2}\right) W_{\bar{k}}=\mathcal{B}_{2}\left(W_{\bar{k}}, W_{\bar{m}}\right)+\nabla p
$$


where $\mathcal{B}_{2}\left(Y^{k}, Y^{m}\right) \in\left\{u \in L^{2}\left(\mathrm{R}_{2}, \mathbb{R}^{2}\right) \mid \partial_{x_{1}} u_{1}+\partial_{x_{2}} u_{2}=0\right.$ and $\left.u \cdot\left(\mathbf{n}_{1}, \mathbf{n}_{2}\right)=0\right\}$ and $p \in$ $H^{1}\left(\mathrm{R}_{2}, \mathbb{R}^{2}\right)$. Therefore from (30) it follows that necessarily

$$
\mathcal{B}\left(Y^{k}, Y^{m}\right)=\left(\begin{array}{c}
\mathcal{B}_{2}\left(W_{\bar{k}}, W_{\bar{m}}\right) \\
0
\end{array}\right) \text { and } \nabla q=\left(\begin{array}{c}
\nabla_{2} p \\
0
\end{array}\right)
$$

Notice that given $x \in \partial \mathrm{R}$, the normal $\mathbf{n}_{x}$, to $\mathrm{R}$ at $x$, satisfies

$\mathbf{n}_{x}=\left(\mathbf{n}_{x, 1}, \mathbf{n}_{x, 2}, \mathbf{n}_{x, 3}\right)=\left(\mathbf{n}_{x, 1}, \mathbf{n}_{x, 2}, 0\right)$ if $x=:\left(\bar{x}, x_{3}\right) \in \partial \mathrm{R}_{2} \times\left(0, L_{3}\right)$, and $\mathbf{n}_{x}=(0,0, \pm 1)$ if $x \in \mathrm{R}_{2} \times\left\{0, L_{3}\right\}$. Notice also that $\partial \mathrm{R}_{2} \times\left(0, L_{3}\right) \cup \mathrm{R}_{2} \times\left\{0, L_{3}\right\}$ is dense in $\partial \mathrm{R}$.

From the results in [25, Section 6.3] (see also [23, Section 7.1], for (B, D $(A))$-saturating sets) we know that if for all $q \geq 3$ and $n \in \mathcal{S}^{q+2}$, with $n_{3}=0$ and $\left(n_{1}, n_{2}\right) \neq(q+2, q+2)$, we have that $W_{\bar{n}} \in \mathcal{G}^{q+2-3+1}$, then for all $n \in \mathcal{S}^{q+1}$, with $n_{3}=0$, we have that $W_{\bar{n}} \in \mathcal{G}^{q}$. Repeating the argument for the cases $n_{1}=0$ and $n_{2}=0$, we arrive at

$$
\operatorname{span}\left\{Y^{n} \mid n \in \mathcal{S}^{q+1}, \#_{0}(n)=1\right\} \subseteq \mathcal{G}^{q} .
$$

- The case $n \in \mathcal{C}^{q+1}$ and $\#_{0}(n)=0$. In this case $n \in \mathbb{N}_{0}^{3}$. We start by defining, again for $q \geq 3$ and for some given $m, m_{1}$, and $m_{2}$ in $\{1,2,3\}$, the index sets

$$
\begin{aligned}
\mathcal{R}_{m}^{q} & :=\left\{n \in \mathcal{S}^{q} \mid n_{m}=q, 1 \leq n_{i} \leq q-1 \text { for } i \neq m\right\}, \\
\mathcal{L}_{m_{1}, m_{2}}^{q} & :=\left\{n \in \mathcal{S}^{q} \mid n_{m_{1}}=q=n_{m_{2}}, m_{1} \neq m_{2}, 1 \leq n_{i} \leq q-1, i \notin\left\{m_{1}, m_{2}\right\}\right\} .
\end{aligned}
$$

We define the set of eigenfunctions

$$
\mathcal{C}_{0}^{q}=\left\{Y^{1, n}, Y^{2, n} \mid n \in \mathcal{S}^{q}, \#_{0}(n)=0\right\} .
$$

Notice that

$$
\begin{aligned}
\left\{n \in \mathcal{S}^{q+1} \mid \#_{0}(n)=0\right\}= & \left\{n \in \mathcal{S}^{q} \mid \#_{0}(n)=0\right\} \bigcup\left(\mathcal{R}_{1}^{q+1} \cup \mathcal{R}_{2}^{q+1} \cup \mathcal{R}_{3}^{q+1}\right) \\
& \bigcup\left(\mathcal{L}_{1,2}^{q+1} \cup \mathcal{L}_{2,3}^{q+1} \cup \mathcal{L}_{3,1}^{q+1}\right) \bigcup\{(q+1, q+1, q+1)\} .
\end{aligned}
$$

It remains to prove that $\mathcal{C}_{0}^{q+1} \subset \mathcal{G}^{q}$, which is a corollary of the following Lemmas 3.4, 3.5, and 3.6 which we will prove in the following Sections 3.5.1, 3.5.2, and 3.5.3.

Lemma 3.4. $Y^{j(n), n} \in \mathcal{G}^{q}$ for all $n \in \bigcup_{i=1}^{3} \mathcal{R}_{i}^{q+1}$.

Lemma 3.5. $Y^{j(n), n} \in \mathcal{G}^{q}$ for all $n \in \mathcal{L}_{1,2}^{q+1} \cup \mathcal{L}_{2,3}^{q+1} \cup \mathcal{L}_{3,1}^{q+1}$.

Lemma 3.6. $Y^{\{1,2\},(q+1, q+1, q+1)} \subset \mathcal{G}^{q}$.

Observe that, from (31) and Lemmas 3.4, 3.5, and 3.6, it follows that

$$
Y^{j(n), n} \in \mathcal{G}^{q} \text { for all } n \in \mathcal{S}^{q+1} .
$$

which implies that $\mathcal{C}^{q+1} \subseteq \mathcal{G}^{q}$. Therefore, we have just proven that (IH.R-eq.29) implies that $\mathcal{C}^{q+1} \subseteq \mathcal{G}^{(q+1)-1}$. Then by induction, using (28), it follows that (27) holds true, which implies the statement of Theorem 3.1 .

3.5.1. Proof of Lemma 3.4. We proceed into 2 main steps:

- Step 1: Generating $Y^{j(n), n}$ with $n \in\{(1, l, q+1),(l, 1, q+1) \mid 0<l \leq q\}$.

- Step 2: Generating $Y^{j(n), n}$ with $n \in\left\{\left(n_{1}, n_{2}, q+1\right) \mid 2 \leq n_{1} \leq q\right.$ and $\left.2 \leq n_{2} \leq q\right\}$.

- Step 1: Generating the family $Y^{j(n), n}$ with $n=(1, l, q+1)$ or $n=(l, 1, q+1)$. We start with $n=(1, l, q+1)$ and proceed by induction on $l$.

Base step. We will prove that

$$
Y^{\{1,2\},(1,1, q+1)} \subset \mathcal{G}^{q} .
$$

To generate $n=(1,1, q+1)$ we choose

$$
\begin{aligned}
k & =(1,0, q), & m & =(0,1,1), \\
w^{k} & =\left(L_{1} q, 0,-L_{3}\right), & w^{m} & =\left(0, L_{2},-L_{3}\right),
\end{aligned}
$$


From (16), this choice gives us

$$
\left(Y^{k} \cdot \nabla\right) Y^{m}+\left(Y^{m} \cdot \nabla\right) Y^{k}=\mathcal{Y}_{z_{\alpha^{1}}}^{(1,1, q+1)}+\mathcal{Y}_{z_{\alpha^{2}}}^{(1,1, q-1)},
$$

for suitable $z_{\alpha^{1}}, z_{\alpha^{2}} \in \mathbb{R}^{3}$. By the induction hypothesis in assumption

IH.R-eq.29, we have $Y^{\{1,2\},(1,1, q-1)}:=\left\{Y^{1,(1,1, q-1)}, Y^{2,(1,1, q-1)}\right\} \subseteq \mathcal{G}^{q-1} \subseteq \mathcal{G}^{q}$, which implies that $\Pi \mathcal{Y}_{z^{\alpha^{2}}}^{(1,1, q-1)} \in \mathcal{G}^{q}$. Hence, we can conclude that $\Pi \mathcal{Y}_{z^{\alpha^{1}}}^{(1,1, q+1)} \in \mathcal{G}^{q}$. Next, we can compute the vector $z_{\alpha^{1}}$ as follows: from

$$
\beta_{w^{k}, m}^{\star_{1} \star_{2}+}=-\frac{\pi}{8}, \quad \beta_{w^{k}, m}^{\star_{1} \star_{2}-}=\frac{\pi}{8}, \quad \beta_{w^{m}, k}^{\star_{1} \star_{2}+}=-\frac{\pi}{8} q, \quad \beta_{w^{m}, k}^{\star_{1} \star_{2}-}=\frac{\pi}{8} q,
$$

with $\left(\star_{1}, \star_{2}\right) \in\{+,-\}^{2}$, we get

$$
\begin{aligned}
z_{\alpha^{1}} & =\left(\begin{array}{c}
0+L_{1} q\left(\beta_{w^{m}, k}^{+++}-\beta_{w^{m}, k}^{++-}+\beta_{w^{m}, k}^{+-+}-\beta_{w^{m}, k}^{+--}\right) \\
L_{2}\left(\beta_{w^{k}, m}^{+++}+\beta_{w^{k}, m}^{++-} \operatorname{sign}(0-1)-\beta_{w^{k}, m}^{+-} \operatorname{sign}(0-1)-\beta_{w^{k}, m}^{+--}\right)+0 \\
-L_{3}\left(\beta_{w^{k}, m}^{+++}+\beta_{w^{k}, m}^{+-+}-\beta_{w^{k}, m}^{+--}-\beta_{w^{k}, m}^{++-}\right)-L_{3}\left(\beta_{w^{m}, k}^{+++}+\beta_{w^{m}, k}^{+-+}-\beta_{w^{m}, k}^{+--}-\beta_{w^{m}, k}^{++-}\right)
\end{array}\right) \\
= & \frac{\pi}{2}\left(\begin{array}{c}
-L_{1} q^{2} \\
L_{2} \\
L_{3}(q+1)
\end{array}\right),
\end{aligned}
$$

Remark 3.1. The factors $\operatorname{sign}(0-1)=\operatorname{sign}\left(k_{2}-m_{2}\right)$ appearing in (3.5.1) are due to the fact that the vector functions $\mathcal{Y}_{z}^{n}$ in (19) are defined for nonnegative frequencies $n \in \mathbb{N}^{3}$, and in (16) the frequencies may be negative. To guarantee nonnegative frequencies we can just rewrite (16) by replacing each $\mathrm{S}_{i}\left(k_{i}-m_{i}\right)$ by its equivalent $\operatorname{sign}\left(k_{i}-m_{i}\right) \mathrm{S}_{i}\left(\left|k_{i}-m_{i}\right|\right)$. Also, recall that $\mathrm{C}_{i}\left(\left|k_{i}-m_{i}\right|\right)=\mathrm{C}_{i}\left(k_{i}-m_{i}\right)$.

Next, we choose

$$
\begin{aligned}
k & =(1,0, q-1), & m & =(0,1,2), \\
w^{k} & =\left(L_{1}(q-1), 0,-L_{3}\right), & w^{m} & =\left(0,2 L_{2},-L_{3}\right),
\end{aligned}
$$

which gives us

$$
\left(Y^{k} \cdot \nabla\right) Y^{m}+\left(Y^{m} \cdot \nabla\right) Y^{k}=\mathcal{Y}_{z^{1}}^{(1,1, q+1)}+\mathcal{Y}_{z_{\gamma^{2}}}^{(1,1, q-2)},
$$

for suitable $z_{\gamma^{1}}, z_{\gamma^{2}} \in \mathbb{R}^{3}$. Again from assumption (IH.R-eq.29) we have $Y^{\{1,2\},(1,1, q-2)} \subseteq \mathcal{G}^{q-1}$, and we can conclude that $\Pi \mathcal{Y}_{z^{1}}^{(1,1, q+1)} \in \mathcal{G}^{q}$. From (16) we find

$$
z_{\gamma^{1}}=\frac{\pi}{2}\left(\begin{array}{c}
-L_{1}(q-1)^{2} \\
-4 L_{2} \\
L_{3}(q+1)
\end{array}\right) .
$$

In order to use Lemma 3.3, we observe that the family $\left\{z_{\alpha^{1}}, z_{\gamma^{1}},(1,1, q+1)\right\}$ in linearly independent, which follows from

$$
\begin{aligned}
& \operatorname{det}\left(n z_{\alpha^{1}} z_{\gamma^{1}}\right)=\frac{\pi^{2}}{4} \operatorname{det}\left(\begin{array}{ccc}
1 & -L_{1} q^{2} & -L_{1}(q-1)^{2} \\
1 & -L_{2} & -4 L_{2} \\
q+1 & L_{3}(q+1) & L_{3}(q+1)
\end{array}\right) \\
& =\frac{\pi^{2}}{4}(q+1)\left(L_{1}\left(L_{2}+L_{3}\right)(2 q-1)+3 L_{1} L_{2} q^{2}+3 L_{2} L_{3}\right)>0 .
\end{aligned}
$$

Therefore Lemma 3.3 give us

$$
Y^{\{1,2\},(1,1, q+1)} \subseteq \mathcal{G}^{q}
$$

Induction step. Now let us assume that

$$
Y^{\{1,2\},(1, l-2, q+1)} \subseteq \mathcal{G}^{q}, \quad \text { for a given } l, \quad 2 \leq l \leq q .
$$

(IH.R[1-eq.37)

Notice that (31) and (36) give us

$$
Y^{\{1,2\},(1, l, q+1)} \subseteq \mathcal{G}^{q}, \quad \text { for all } \quad l \in\{0,1\} .
$$


In order to generate $Y^{\{1,2\},(1, l, q+1)}$ we choose

$$
\begin{aligned}
k & =(1, l-1, q), & m & =(0,1,1), \\
w^{k} & =\left(0, L_{2} q, L_{3}(1-l)\right), & w^{m} & =\left(0, L_{2},-L_{3}\right),
\end{aligned}
$$

This choice gives us

$$
\left(Y^{k} \cdot \nabla\right) Y^{m}+\left(Y^{m} \cdot \nabla\right) Y^{k}=\mathcal{Y}_{z_{\alpha^{1}}}^{(1, l, q+1)}+\mathcal{Y}_{z_{\alpha^{2}}}^{(1, l-2, q+1)}+\mathcal{Y}_{z_{\alpha^{3}}}^{(1, l, q-1)}+\mathcal{Y}_{z_{\alpha^{3}}}^{(1, l-2, q-1)} .
$$

From assumption (IH.R-eq.29) we have that both $Y^{j(1, l, q-1),(1, l, q-1)}$ and $Y^{j(1, l-2, q-1),(1, l-2, q-1)}$ belong to $\mathcal{G}^{q-1}$; and from assumption (IH.R1teq.37) we have $Y^{j(1, l-2, q+1),(1, l-2, q+1)} \in$ $\mathcal{G}^{q}$. Thus, we can conclude that $\Pi \mathcal{Y}_{z_{\alpha}}^{(1, l, q+1)} \in \mathcal{G}^{q}$.

To compute $z_{\alpha^{1}}$ we use

$$
\beta_{w^{k}, m}^{+++}=\beta_{w^{k}, m}^{-++}=\frac{\pi}{8}(q-l+1) \quad \text { and } \quad \beta_{w^{m}, k}^{+++}=\beta_{w^{m}, k}^{-++}=\frac{\pi}{8}(l-q-1),
$$

and obtain

$$
\begin{aligned}
z_{\alpha^{1}} & =\left(\begin{array}{c}
0 \\
L_{2}\left(\beta_{w^{k}, m}^{+++}+\beta_{w^{k}, m}^{-++}\right)+L_{2} q\left(\beta_{w^{m}, k}^{+++}+\beta_{w^{m}, k}^{-++}\right) \\
-L_{3} \cdot\left(\beta_{w^{k}, m}^{++}+\beta_{w^{k}, m}^{-++}\right)+L_{3}(1-l) \cdot\left(\beta_{w^{m}, k}^{+++}+\beta_{w^{m}, k}^{-++}\right)
\end{array}\right) \\
& =\frac{\pi}{4}\left(\begin{array}{c}
0 \\
L_{2}(q-l+1)(1-q) \\
L_{3}(q-l+1)(l-2)
\end{array}\right) .
\end{aligned}
$$

Next, we choose the same frequencies $(k, m)$ with different $\left(w^{k}, w^{m}\right)$ :

$$
\begin{aligned}
k & =(1, l-1, q), & m & =(0,1,1), \\
w^{k} & =\left(L_{1} q, 0,-L_{3}\right), & w^{m} & =\left(0, L_{2},-L_{3}\right),
\end{aligned}
$$

Proceeding as above, we obtain that $\Pi \mathcal{Y}_{z_{\gamma} 1}^{(1, l, q+1)} \in \mathcal{G}^{q}$ and, from

we find

$$
\beta_{w^{k}, m}^{+++}=\beta_{w^{k}, m}^{-++}=-\frac{\pi}{8} \quad \text { and } \quad \beta_{w^{m}, k}^{+++}=\beta_{w^{m}, k}^{-++}=\frac{\pi}{8}(l-q-1),
$$

$$
z_{\gamma^{1}}=\frac{\pi}{4}\left(\begin{array}{c}
-L_{1} q(q-l+1) \\
-L_{2} \\
L_{3}(q-l+2)
\end{array}\right)
$$

Then, from

$$
\begin{aligned}
\frac{16}{\pi^{2}} \operatorname{det}\left(n z_{\alpha^{1}} z_{\gamma^{1}}\right)= & \operatorname{det}\left(\begin{array}{ccc}
1 & 0 & -L_{1} q(q-l+1) \\
l & L_{2}(q-l+1)(1-q) & -L_{2} \\
q+1 & L_{3}(q-l+1)(l-2) & L_{3}(q-l+2)
\end{array}\right) \\
= & \operatorname{det}\left(\begin{array}{cc}
L_{2}(q-l+1)(1-q) & -L_{2} \\
L_{3}(q-l+1)(l-2) & L_{3}(q-l+2)
\end{array}\right) \\
& -L_{1} q(q-l+1) \operatorname{det}\left(\begin{array}{cc}
l & L_{2}(q-l+1)(1-q) \\
q+1 & L_{3}(q-l+1)(l-2)
\end{array}\right) \\
= & -L_{2} L_{3} q(q-l+1)^{2}-L_{1} q(q-l+1)^{2}\left(L_{3} l^{2}-2 L_{3} l+L_{2} q^{2}-L_{2}\right) \\
= & -q(q-l+1)^{2}\left[L_{2} L_{3}+L_{1} L_{3} l(l-2)+L_{1} L_{2}\left(q^{2}-1\right)\right]<0,
\end{aligned}
$$

since $2 \leq l \leq q$, using Lemma 3.3. we can conclude that $Y^{\{1,2\},(1, l, q+1)} \in \mathcal{G}^{q}$.

We have just proven that assumption (IH.R1+eq.37) leads us to $Y^{\{1,2\},(1, l, q+1)} \in \mathcal{G}^{q}$. Then by induction, using (38), we can conclude that

$$
\left\{Y^{\{1,2\},(1, l, q+1)} \mid 0<l \leq q\right\} \subseteq \mathcal{G}^{q},
$$


and by a similar argument we can derive that

$$
\left\{Y^{\{1,2\},(l, 1, q+1)} \mid 0<l \leq q\right\} \subseteq \mathcal{G}^{q} .
$$

- Step 2. Generating the family $Y^{j(n), n}$ with $n=\left(n_{1}, n_{2}, q+1\right)$ where $2 \leq n_{1} \leq q$ and $2 \leq n_{2} \leq q$. Again, we proceed by induction on the pair $\left(n_{1}, n_{2}\right)$, under the lexicographical order $\left(n_{1}, n_{2}\right)<$ $\left(m_{1}, m_{2}\right)$ iff $n_{1}<m_{1}$, or $n_{1}=m_{1}$ and $n_{2}<m_{2}$, defined on the set $N_{q}:=\left\{\left(\kappa_{1}, \kappa_{2}\right) \in\right.$ $\left.\{0,1,2, \ldots, q\}^{2} \backslash\{0,0\}\right\}$.

Base step. From (31), (38), and (39), we know that

$$
Y^{j(n), n} \in \mathcal{G}^{q}, \quad \text { for all } n=\left(n_{1}, n_{2}, q+1\right), \quad\left(n_{1}, n_{2}\right) \in N_{q}, \quad(0,0)<\left(n_{1}, n_{2}\right)<(2,2) .
$$

Induction step. Now we assume that

$$
Y^{j(\kappa), \kappa} \in \mathcal{G}^{q}, \quad \text { for all } \quad \kappa \in N_{q} \quad \text { with } \quad\left\{\begin{array}{l}
(0,0)<\left(\kappa_{1}, \kappa_{2}\right)<\left(n_{1}, n_{2}\right) \leq(q, q), \\
(2,2) \leq\left(n_{1}, n_{2}\right), \quad \kappa_{3}=q+1
\end{array}\right.
$$

(IH.R[1-eq.41)

We want to prove that $Y^{j(n), n} \in \mathcal{G}^{q}$, with $n=\left(n_{1}, n_{2}, q+1\right)$.

By choosing

$$
\begin{aligned}
k & =\left(n_{1}-1, n_{2}-1, q\right), & m & =(1,1,1), \\
w^{k} & =\left(0, L_{2} q, L_{3}\left(1-n_{2}\right)\right), & w^{m} & =\left(0, L_{2},-L_{3}\right),
\end{aligned}
$$

we find

$$
\left(Y^{k} \cdot \nabla\right) Y^{m}+\left(Y^{m} \cdot \nabla\right) Y^{k}=\mathcal{Y}_{z_{\alpha}}^{\left(n_{1}, n_{2}, q+1\right)}+\sum_{i=2}^{8} \mathcal{Y}_{z_{\alpha^{i}}}^{\kappa^{i}},
$$

with $\left\{\kappa^{i} \mid 2 \leq i \leq 8\right\}=\left\{\left(n_{1}-2, n_{2}-2, q-1\right),\left(n_{1}, n_{2}-2, q-1\right),\left(n_{1}-2, n_{2}, q-1\right),\left(n_{1}, n_{2}, q-\right.\right.$ 1) $\left.\left(n_{1}-2, n_{2}-2, q+1\right),\left(n_{1}, n_{2}-2, q+1\right),\left(n_{1}-2, n_{2}, q+1\right)\right\}$. From assumption (IH.R-eq.29), we find that $\Pi \mathcal{Y}_{z_{\alpha^{i}}}^{\kappa^{i}} \in \mathcal{G}^{q-1}$, for $\kappa^{i} \in\left\{\left(n_{1}, n_{2}-2, q-1\right),\left(n_{1}-2, n_{2}, q-1\right),\left(n_{1}, n_{2}, q-1\right)\right\}$; and assumption (IH.R[1-eq.41) implies that $\Pi \mathcal{Y}_{z_{\alpha^{i}}}^{\kappa^{i}} \in \mathcal{G}^{q}$, for $\kappa^{i} \in\left\{\left(n_{1}, n_{2}-2, q+1\right),\left(n_{1}-2, n_{2}, q+1\right)\right\}$.

Now if $\left(n_{1}, n_{2}\right)>(2,2)$, then again by assumptions (IH.R-eq.29)

and (IH.R11-eq.41) we find that $\Pi \mathcal{Y}_{z_{\alpha^{i}}}^{\kappa^{i}} \in \mathcal{G}^{q}$, with $\kappa^{i} \in\left\{\left(n_{1}-2, n_{2}-2, q-1\right),\left(n_{1}-2, n_{2}-2, q-1\right)\right\}$. On the other hand if $\left(n_{1}, n_{2}\right)=(2,2)$, then $\Pi \mathcal{Y}_{z_{\alpha^{i}}}^{\kappa^{i}}=0 \in \mathcal{G}^{q}$, with $\kappa^{i} \in\left\{\left(n_{1}-2, n_{2}-2, q-\right.\right.$ $\left.1),\left(n_{1}-2, n_{2}-2, q+1\right)\right\}$.

Thus, we can conclude that $\Pi \mathcal{Y}_{z_{\alpha} 1}^{\left(n_{1}, n_{2}, q+1\right)} \in \mathcal{G}^{q}$. Now, from

$$
\beta_{w^{k}, m}^{+++}=\frac{\pi}{8}\left(q-n_{2}+1\right) \quad \text { and } \quad \beta_{w^{m}, k}^{+++}=\frac{\pi}{8}\left(n_{2}-q-1\right),
$$

we obtain

$$
z_{\alpha^{1}}=\frac{\pi}{8}\left(\begin{array}{c}
0 \\
L_{2}\left(q-n_{2}+1\right)(1-q) \\
L_{3}\left(q-n_{2}+1\right)\left(2-n_{2}\right)
\end{array}\right) .
$$

Analogously with the choice

$$
\begin{aligned}
k & =\left(n_{1}-1, n_{2}-1, q\right), & m & =(1,1,1), \\
w^{k} & =\left(L_{1} q, 0, L_{3}\left(1-n_{1}\right)\right), & w^{m} & =\left(0, L_{2},-L_{3}\right),
\end{aligned}
$$

we can conclude that $\Pi \mathcal{Y}_{z_{\gamma^{1}}}^{\left(n_{1}, n_{2}, q+1\right)} \in \mathcal{G}^{q}$ and, from

$$
\beta_{w^{k}, m}^{+++}=\frac{\pi}{8}\left(q-n_{1}+1\right) \quad \text { and } \quad \beta_{w^{m}, k}^{+++}=\frac{\pi}{8}\left(n_{2}-q-1\right),
$$



we obtain

$$
z_{\gamma^{1}}=\frac{\pi}{8}\left(\begin{array}{c}
-L_{1} q\left(q-n_{2}+1\right) \\
L_{2}\left(q-n_{1}+1\right) \\
L_{3}\left(1-n_{1}\right)\left(q-n_{2}+1\right)+L_{3}\left(q-n_{1}+1\right)
\end{array}\right) .
$$

The family $\left\{n, z_{\alpha^{1}}, z_{\gamma^{1}}\right\}$ is linearly independent, because

$$
\begin{aligned}
& \operatorname{det}\left(\begin{array}{ccc}
n_{1} & 0 & -L_{1} q\left(q-n_{2}+1\right) \\
n_{2} & L_{2}\left(q-n_{2}+1\right)(1-q) & L_{2}\left(q-n_{1}+1\right) \\
q+1 & L_{3}\left(q-n_{2}+1\right)\left(n_{2}-2\right) & L_{3}\left(n_{1}-1\right)\left(q-n_{2}+1\right)-L_{3}\left(q-n_{1}+1\right)
\end{array}\right) \\
= & n_{1}\left(q-n_{2}+1\right) \operatorname{det}\left(\begin{array}{cc}
L_{2}(1-q) & L_{2}\left(q-n_{1}+1\right) \\
L_{3}\left(n_{2}-2\right) & L_{3}\left(n_{1}-1\right)\left(q-n_{2}+1\right)-L_{3}\left(q-n_{1}+1\right)
\end{array}\right) \\
- & L_{1} q\left(q-n_{2}+1\right)^{2} \operatorname{det}\left(\begin{array}{cc}
n_{2} & L_{2}(1-q) \\
q+1 & L_{3}\left(n_{2}-2\right)
\end{array}\right) \\
= & -q\left(q-n_{2}+1\right)^{2}\left[L_{2} L_{3} n_{1}\left(n_{1}-2\right)+L_{1} L_{3} n_{2}\left(n_{2}-2\right)+L_{1} L_{2}\left(q^{2}-1\right)\right]<0,
\end{aligned}
$$

since $2 \leq n_{1} \leq q$ and $2 \leq n_{1} \leq q$. Thus from Lemma 3.3 we have that $Y^{\{1,2\},\left(n_{1}, n_{2}, q+1\right)} \subset \mathcal{G}^{q}$.

We have just proved that assumption (IH.R 1+eq.41) implies that

$$
Y^{\{1,2\},\left(n_{1}, n_{2}, q+1\right)} \in \mathcal{G}^{q} .
$$

Therefore, using (40), by induction it follows that $Y^{\{1,2\}, n} \in \mathcal{G}^{q}$ with $n=\left(n_{1}, n_{2}, q+1\right)$ and $\left(n_{1}, n_{2}\right) \in N_{q}$, which implies that $Y^{\{1,2\}, n} \in \mathcal{G}^{q}$ for all $n \in \mathcal{R}_{3}^{q+1}$. An analogous argument leads us to

$$
Y^{\{1,2\}, n} \in \mathcal{G}^{q}, \quad \text { for all } n \in \mathcal{R}_{1}^{q+1} \cup \mathcal{R}_{2}^{q+1} \cup \mathcal{R}_{3}^{q+1},
$$

which ends the proof of Lemma 3.4.

\subsubsection{Proof of Lemma 3.5. .}

We prove that $Y^{j(n), n} \in \mathcal{G}^{q}$ for $n=(l, q+1, q+1) \in \mathcal{L}_{2,3}^{q+1}, 1 \leq l \leq q$. We choose

$$
\begin{aligned}
k & =(l, q-1, q), & m & =(0,2,1), \\
w^{k} & =\left(0, L_{2} q, L_{3}(1-q)\right), & w^{m} & =\left(0, L_{2},-2 L_{3}\right),
\end{aligned}
$$

which leads us to

$$
\left(Y^{k} \cdot \nabla\right) Y^{m}+\left(Y^{m} \cdot \nabla\right) Y^{k}=\mathcal{Y}_{z_{\alpha^{1}}}^{(l, q+1, q+1)}+\mathcal{Y}_{z_{\alpha^{2}}}^{(l, q-2, q+1)}+\mathcal{Y}_{z_{\alpha^{3}}}^{(l, q+1, q-1)}+\mathcal{Y}_{z_{\alpha^{4}}}^{(l, q-2, q-1)} .
$$

By the induction hypothesis (IH.R-eq.29) we have $\Pi \mathcal{Y}_{z_{\alpha}}^{(l, q-2, q-1)} \in \mathcal{G}^{q-1}$. From (42), since $\{(l, q-2, q+1),(l, q+1, q-1)\} \subset \mathcal{R}_{3}^{q+1} \cup \mathcal{R}_{2}^{q+1}$, we also have $\Pi \mathcal{Y}_{z_{\alpha^{2}}}^{(l, q-2, q+1)}+\Pi \mathcal{Y}_{z_{\alpha^{3}}}^{(l, q+1, q-1)} \in \mathcal{G}^{q}$. Therefore, we obtain that $\Pi \mathcal{Y}_{z_{\alpha}}^{(l, q+1, q+1)} \in \mathcal{G}^{q}$. Now, from

$$
\beta_{w^{k}, m}^{+++}=\beta_{w^{k}, m}^{-++}=\frac{\pi}{8}(q+1) \quad \text { and } \quad \beta_{w^{m}, k}^{+++}=\beta_{w^{m}, k}^{-++}=-\frac{\pi}{8}(q+1),
$$

we obtain

$$
\begin{aligned}
z_{\alpha^{1}} & =\left(\begin{array}{c}
0 \\
L_{2}\left(\beta_{w^{k}, m}^{+++}+\beta_{w^{k}, m}^{-++}\right)+L_{2} q\left(\beta_{w^{m}, k}^{+++}+\beta_{w^{m}, k}^{-++}\right) \\
-2 L_{3}\left(\beta_{w^{k}, m}^{+++}+\beta_{w^{k}, m}^{-++}\right)+L_{3}(1-q)\left(\beta_{w^{m}, k}^{++}+\beta_{w^{m}, k}^{-++}\right)
\end{array}\right) \\
& =\frac{\pi}{4}\left(\begin{array}{c}
0 \\
L_{2}\left(1-q^{2}\right) \\
L_{3}(q+1)(q-2)
\end{array}\right) .
\end{aligned}
$$

Analogously the choice

$$
\begin{aligned}
k & =(l, q-1, q), & m & =(0,2,1), \\
w^{k} & =\left(L_{1} q, 0,-L_{3} l\right), & w^{m} & =\left(0, L_{2},-2 L_{3}\right),
\end{aligned}
$$


allow us to conclude that $\Pi \mathcal{Y}_{z_{\gamma^{1}}}^{(l, q+1, q+1)} \in \mathcal{G}^{q}$. Where, from

$$
\beta_{w^{k}, m}^{+++}=\beta_{w^{k}, m}^{-++}=-\frac{\pi}{8} l \quad \text { and } \quad \beta_{w^{m}, k}^{+++}=\beta_{w^{m}, k}^{-++}=-\frac{\pi}{8}(q+1),
$$

we have

$$
z_{\gamma^{1}}=\frac{\pi}{4}\left(\begin{array}{c}
-L_{1} q(q+1) \\
-L_{2} l \\
L_{3} l(q+3)
\end{array}\right)
$$

Now from Lemma 3.3 and

$$
\begin{aligned}
\operatorname{det}\left(n z_{\alpha^{1}} z_{\gamma^{1}}\right) & =\operatorname{det}\left(\begin{array}{ccc}
l & 0 & -L_{1} q(q+1) \\
q+1 & L_{2}\left(1-q^{2}\right) & -L_{2} l \\
q+1 & L_{3}(q+1)(q-2) & L_{3} l(q+3)
\end{array}\right) \\
& =-q(q+1)^{2}\left[L_{2} L_{3} l^{2}+L_{1} L_{3}(q+1)(q-2)+L_{1} L_{2}\left(q^{2}-1\right)\right]<0,
\end{aligned}
$$

because $l \geq 1$ and $q \geq 3$, it follows that $Y^{\{1,2\},(l, q+1, q+1)} \in \mathcal{G}^{q}$, for $1 \leq l \leq q$. A similar argument gives us

$$
Y^{\{1,2\}, n} \in \mathcal{G}^{q}, \quad \text { for all } n \in \mathcal{L}_{1,2}^{q+1} \cup \mathcal{L}_{2,3}^{q+1} \cup \mathcal{L}_{3,1}^{q+1},
$$

which ends the proof of Lemma 3.5.

3.5.3. Proof of Lemma 3.6. Firstly, we choose

$$
\begin{aligned}
k & =(q, q-1, q), & m & =(1,2,1), \\
w^{k} & =\left(0, L_{2} q, L_{3}(1-q)\right), & w^{m} & =\left(0, L_{2},-2 L_{3}\right),
\end{aligned}
$$

which give us

$$
\left(Y^{k} \cdot \nabla\right) Y^{m}+\left(Y^{m} \cdot \nabla\right) Y^{k}=\mathcal{Y}_{z_{\alpha^{1}}}^{(q+1, q+1, q+1)}+\sum_{i=2}^{8} \mathcal{Y}_{z_{\alpha^{i}}}^{\kappa^{i}},
$$

where $\left\{\kappa^{i} \mid i \in\{2, \cdots, 8\}\right\}=\{(q+1, q+1, q-1),(q-1, q+1, q+1)),(q-1, q-2, q+1),(q-$ $1, q+1, q-1),(q+1, q-2, q-1),(q-1, q-2, q+1),(q-1, q-2, q-1)\}$.

Since

$$
\left\{\kappa^{i} \mid i \in\{2, \cdots, 8\}\right\} \subseteq\left(\mathcal{R}_{1}^{q+1} \cup \mathcal{R}_{2}^{q+1} \cup \mathcal{R}_{3}^{q+1}\right) \bigcup\left(\mathcal{L}_{1,2}^{q+1} \cup \mathcal{L}_{2,3}^{q+1}\right) \bigcup \mathcal{S}^{q}
$$

from (IH.R-eq.29), (42), and (43) we can conclude that $\Pi \mathcal{Y}_{z_{\alpha^{1}}}^{(q+1, q+1, q+1)} \in \mathcal{G}^{q}$.

Now, from the identities

$$
\beta_{w^{k}, m}^{+++}=\frac{\pi}{8}(q+1) \quad \text { and } \quad \beta_{w^{m}, k}^{+++}=-\frac{\pi}{8}(q+1),
$$

we obtain

$$
z_{\alpha^{1}}=\left(\begin{array}{c}
0 \\
L_{2} \cdot \beta_{w^{k}, m}^{+++}+L_{2} q \cdot \beta_{w^{m}, k}^{+++} \\
-2 L_{3} \cdot \beta_{w^{k}, m}^{+++}+L_{3}(1-q) \cdot \beta_{w^{m}, k}^{+++}
\end{array}\right)=\frac{\pi}{8}\left(\begin{array}{c}
0 \\
L_{2}\left(1-q^{2}\right) \\
L_{3}(q+1)(q-2)
\end{array}\right) .
$$

Next by choosing

$$
\begin{aligned}
k & =(q, q-1, q), & m & =(1,2,1), \\
w^{k} & =\left(L_{1}(1-q), L_{2} q, 0\right), & w^{m} & =\left(0, L_{2},-2 L_{3}\right),
\end{aligned}
$$

and proceeding as above, we can conclude that $\Pi \mathcal{Y}_{z_{\gamma^{1}}}^{(q+1, q+1, q+1)} \in \mathcal{G}^{q}$, with

$$
z_{\gamma^{1}}=\left(\begin{array}{c}
0 \beta_{w^{k}, m}^{+++}+L_{1}(1-q) \beta_{w^{m}, k}^{+++} \\
L_{2} \beta_{w^{k}, m}^{+++}+L_{2} q \beta_{w^{m}, k}^{+++} \\
-2 L_{3} \cdot \beta_{w^{k}, m}^{+++}+0 \beta_{w^{m}, k}^{+++}
\end{array}\right)=\frac{\pi}{8}\left(\begin{array}{c}
L_{1}\left(q^{2}-1\right) \\
L_{2}\left(1-q^{2}\right) \\
-2 L_{3}(q+1)
\end{array}\right) .
$$


With $n=(q+1, q+1, q+1)$, using again Lemma 3.3 and

$$
\begin{aligned}
& \operatorname{det}\left(n z_{\alpha^{1}} z_{\gamma^{1}}\right)=\frac{\pi^{2}}{64}(q+1)^{3} \operatorname{det}\left(\begin{array}{ccc}
1 & 0 & L_{1}(q-1) \\
1 & L_{2}(1-q) & L_{2}(1-q) \\
1 & L_{3}(q-2) & -2 L_{3}
\end{array}\right) \\
& \quad=\frac{\pi^{2}}{64}(q+1)^{3}\left[L_{2} L_{3} \operatorname{det}\left(\begin{array}{cc}
1-q & 1-q \\
q-2 & -2
\end{array}\right)+L_{1}(q-1) \operatorname{det}\left(\begin{array}{cc}
1 & L_{2}(1-q) \\
1 & L_{3}(q-2)
\end{array}\right)\right] \\
& \quad=\frac{\pi^{2}}{64}(q+1)^{3}(q-1)\left[\left(L_{1} L_{2}+L_{2} L_{3}\right)(q-1)+L_{1} L_{3}(q-2)\right]>0,
\end{aligned}
$$

because $q \geq 3$, we obtain

$$
Y^{\{1,2\},(q+1, q+1, q+1)} \subset \mathcal{G}^{q},
$$

which ends the proof of Lemma 3.6.

\section{Final Remarks}

We proved the approximate controllability of the Navier-Stokes system in a 3D rectangle by degenerate (low modes) forcing, under Lions boundary conditions. We used the analogous 2D result, derived in [25] (see also 23] for (B, D $(A)$ )-saturating sets). In [20] the case of a 2D cylinder is considered, thus we may wonder whether we can also derive the approximate controllability for the case of a $3 \mathrm{D}$ cylinder. This case can be seen as the case where the fluid is contained in a long (infinite) 3D channel with Lions boundary conditions, and with the periodicity assumption on the long (infinite) direction, thus it is a case of interest for applications. First computations show that the existence of a ( $\mathrm{L}, \mathrm{D}(A))$-saturating set in this case is plausible, but the computations details are still to be checked. Since those computations will be long, and since this manuscript is already long, we will investigate the case of a 3D cylinder in a future work.

We underline that the presented saturating set is (by definition) independent of the viscosity coefficient $\nu$. That is, approximate controllability holds by means of controls taking values in $\mathcal{G}^{1}=\operatorname{span}(\mathcal{C})+\operatorname{span} \mathcal{B}(\mathcal{C}, \operatorname{span} \mathcal{C})=\operatorname{span}(\mathcal{C} \cup \mathcal{B}(\mathcal{C}, \mathcal{C}))$, for any $\nu>0$. It is plausible that a $(\mathrm{L}, \mathrm{D}(A)$ )-saturating set with less elements does exist, but it is not our goal here to minimize the number of elements of $\mathcal{C}$.

We have used the result in [28] where it is proven that under Dirichlet boundary conditions the existence of a $(\mathrm{B}, \mathrm{D}(A))$-saturating set implies the approximate controllability of Navier-Stokes system by degenerate forcing. We can conclude from our results that the same controllability result follows from the existence of a ( $\mathrm{L}, \mathrm{D}(A))$-saturating set. However, up to our knowledge, neither the existence of a $(\mathrm{B}, \mathrm{D}(A))$-saturating set nor that of a $(\mathrm{L}, \mathrm{D}(A))$-saturating set is known under Dirichlet boundary conditions. That is, essentially the approximate controllability of the Navier-Stokes system is still an open problem under Dirichlet boundary conditions. Therefore, it is of interest to find a saturating set for such classical boundary conditions, because they are the most realistic in many situations.

Up to now the known examples of saturating sets consist of eigenfuntions of the Stokes operator. For applications, it would be interesting to consider more realistic functions as actuators, as locally supported functions, recall [1, Problem VII] (cf. [20, Section V]). Furthermore, the explicit expressions for the Stokes operator may be not available as it is the case (up to our best knowledge) for Dirichlet boundary conditions. Here, and in previous works the density of $\bigcup_{j \in \mathbb{N}} \mathcal{G}^{j}$ in $H$ has been proven by showing the the union contains all the eigenfunctions of the Stokes operator. Thus this argument may be difficult (maybe, not possible) to use in the case of Dirichlet boundary conditions, which makes the investigation of these last boundary conditions an interesting problem.

Acknowledgments. The authors acknowledge partial support from the Austrian Science Fund (FWF): P 26034-N25. D. Phan appreciates partial support from the Foundation of Tampere University of Technology. 


\section{REFERENCES}

[1] A. A. Agrachev. Some open problems. arXiv:1304.2590v2 [math.OC] 12 Apr 2013. URL: http://arxiv.org/abs/1304.2590v2

[2] A. A. Agrachev, S. Kuksin, A. V. Sarychev, and A. Shirikyan. On finite-dimensional projections of distributions for solutions of randomly forced 2D Navier-Stokes equations. Ann. Inst. H. Poincaré Probab. Statist., 43(4):399-415, 2007. doi:10.1016/j.anihpb.2006.06.001.

[3] A. A. Agrachev and A. V. Sarychev. Navier-Stokes equations: Controllability by means of low modes forcing. J. Math. Fluid Mech., 7(1):108-152, 2005. doi:10.1007/s00021-004-0110-1

[4] A. A. Agrachev and A. V. Sarychev. Controllability of 2D Euler and Navier-Stokes equations by degenerate forcing. Commun. Math. Phys., 265(3):673-697, 2006. doi:10.1007/s00220-006-0002-8.

[5] A. A. Agrachev and A. V. Sarychev. Solid controllability in fluid dynamics. In Instability in Models Connected with Fluid Flow I, volume 6 of International Mathematical Series, pages 1-35 (ch. 1). Springer, 2008. doi:10.1007/978-0-387-75217-4_1.

[6] R. Aris. Vectors, Tensors, and the Basic Equations of Fluid Mechanics. Dover, New York, 1989. Reprint of the Prentice-Hall 1962 edition. URL: http://store.doverpublications.com/0486661105.html

[7] T. Chambrion, P. Mason, M. Sigalotti, and U. Boscain. Controllability of the discrete-spectrum Schrödinger equation driven by an external field. Ann. Inst. H. Poincaré Anal. Non Linéaire, 26(1):329-349, 2009. doi:10.1016/j.anihpc.2008.05.001

[8] N. V. Chemetov, F. Cipriano, and S. Gavrilyuk. Shallow water model for lakes with friction and penetration. Math. Meth. Appl. Sci., 33(6):687-703, 2010. doi:10.1002/mma.1185.

[9] J. I. Diaz. Obstruction and some approximate controllability results for the Burgers equation and related problems. 174:63-76, 1996.

[10] W. E and J. C. Mattingly. Ergodicity for the Navier-Stokes equation with degenerate random forcing: Finite dimensional approximation. Comm. Pure Appl. Math., 54(11):1386-1402, 2001. doi:10.1002/cpa.10007.

[11] E. Fernández-Cara and S. Guerrero. Null controllability of the Burgers system with distributed controls. Systems Control Lett., 56(5):366-372, 2007. doi:10.1016/j.sysconle.2006.10.022.

[12] M. Hairer and J. C. Mattingly. Ergodicity of the 2D Navier-Stokes equations with degenerate stochastic forcing. Ann. Math., 164(3):993-1032, 2006. doi:10.4007/annals.2006.164.993

[13] A. A. Ilyin and E. S. Titi. Sharp estimates for the number of degrees of freedom for the damped-driven 2-D Navier-Stokes equations. J. Nonlinear Sci., 16(3):233-253, 2006. doi:10.1007/s00332-005-0720-7.

[14] J. P. Kelliher. Navier-Stokes equations with Navier boundary conditions for a bounded domain in the plane. SIAM J. Math. Anal., 38(1):210-232, 2006. doi:10.1137/040612336.

[15] J.-L. Lions. Quelques Méthodes de Résolution des Problèmes aux Limites Non Linéaires. Dunod et GauthierVillars, Paris, 1969.

[16] V. Nersesyan. Approximate controllability of Lagrangian trajectories of the 3D Navier-Stokes system by a finite-dimensional force. Nonlinearity, 28(3):825-848, 2015. doi:10.1088/0951-7715/28/3/825.

[17] H. Nersisyan. Controllability of 3D incompressible Euler equations by a finite-dimensional external force. ESAIM Control, Optim. Calc. Var., 16(3):677-694, 2010. doi:10.1051/cocv/2009017

[18] H. Nersisyan. Controllability of the 3D compressible Euler system. Comm. Partial Differential Equations, 36(9):1544-1564, 2011. doi:10.1080/03605302.2011.596605

[19] D. Phan. Stabilization to Trajectories and Approximate Controllability for the Equations of Fluid Mechanics. PhD Thesis. J. Kepler Universität Linz, Austria, 2016. URL: http://epub.jku.at/obvulihs/content/titleinfo/1592928.

[20] D. Phan and S. S. Rodrigues. Approximate controllability for equations of fluid mechanics with a few body controls. In Proceedings of the 2015 European Control Conference (ECC), Linz, Austria, pages 2682-2687, July 2015. doi:10.1109/ECC.2015.7330943

[21] D. Phan and S. S. Rodrigues. Gevrey regularity for Navier-Stokes equations under Lions boundary conditions. J. Funct. Anal., 272(7):2865-2898, 2017. doi:10.1016/j.jfa.2017.01.014.

[22] S. S. Rodrigues. Controllability issues for the Navier-Stokes equation on a Rectangle. In Proceedings 44th IEEE CDC-ECC'05, Seville, Spain, pages 2083-2085, December 2005. doi:10.1109/CDC.2005.1582468

[23] S. S. Rodrigues. Navier-Stokes equation on the Rectangle: Controllability by means of low modes forcing. J. Dyn. Control Syst., 12(4):517-562, 2006. doi:10.1007/s10883-006-0004-z.

[24] S. S. Rodrigues. Controllability of nonlinear pdes on compact Riemannian manifolds. In Proceedings WMCTF'07; Lisbon, Portugal, pages 462-493, April 2007. URL: http://people.ricam.oeaw.ac.at/s.rodrigues/.

[25] S. S. Rodrigues. Methods of Geometric Control Theory in Problems of Mathematical Physics. PhD Thesis. Universidade de Aveiro, Portugal, 2008. URL: http://hdl.handle.net/10773/2931.

[26] M. Romito. Ergodicity of the finite dimensional approximation of the 3D Navier-Stokes equations forced by a degenerate noise. J. Stat. Phys., 114(1/2):155-177, 2004. doi:10.1023/B: JOSS.0000003108.92097.5c

[27] A. Sarychev. Controllability of the cubic Schroedinger equation via a low-dimensional source term. Math. Control Relat. Fields, 2(3):247-270, 2012. doi:10.3934/mcrf.2012.2.247.

[28] A. Shirikyan. Approximate controllability of three-dimensional Navier-Stokes equations. Comm. Math. Phys., 266(1):123-151, 2006. doi:10.1007/s00220-006-0007-3. 
[29] A. Shirikyan. Controllability of nonlinear PDEs: Agrachev-Sarychev approach. Journées Équations aux Dérivées Partielles. Évian, 4 juin-8 juin. Exposé no. IV, pages 1-11, 2007. URL: https://eudml.org/doc/10631.

[30] A. Shirikyan. Exact controllability in projections for three-dimensional Navier-Stokes equations. Ann. Inst. H. Poincaré Anal. Non Linéaire, 24(4):521-537, 2007. doi:10.1016/j .anihpc.2006.04.002.

[31] A. Shirikyan. Euler equations are not exactly controllable by a finite-dimensional external force. Physica $D$, 237(10-11):1317-1323, 2008. doi:10.1016/j.physd.2008.03.021.

[32] A. Shirikyan. Global exponential stabilisation for the burgers equation with localised control. J. Éc. polytech. Math., 4:613-632, 2017. doi:10.5802/jep.53.

[33] R. Temam. Navier-Stokes Equations and Nonlinear Functional Analysis. Number 66 in CBMS-NSF Regional Conf. Ser. Appl. Math. SIAM, Philadelphia, 2nd edition, 1995. doi:10.1137/1.9781611970050

[34] Y. Xiao and Z. Xin. On the vanishing viscosity limit for the 3D Navier-Stokes equations with a slip boundary condition. Comm. Pure Appl. Math., 60(7):1027-1055, 2007. doi:10.1002/cpa.20187.

[35] Y. Xiao and Z. Xin. On the inviscid limit of the 3D Navier-Stokes equations with generalized Navier-slip boundary conditions. Commun. Math. Stat., 1(3):259-279, 2013. doi:10.1007/s40304-013-0014-6

DUY PHAN

Mathematics Laboratory, Tampere University of Technology,

P.O. Box 553, 33101 TAMPere, Finland.

E-MAIL: duy.phan-duc@tut.fi

SÉRgIO S. RODRIGUES

Johann Radon Institute for Computational and Applied Mathematics, ÖAW,

Altenbergerstraße 69, 4040 Linz, Austria. Tel. +43 7322468 5241,

E-MAIL: sergio.rodrigues@ricam.oeaw.ac.at 